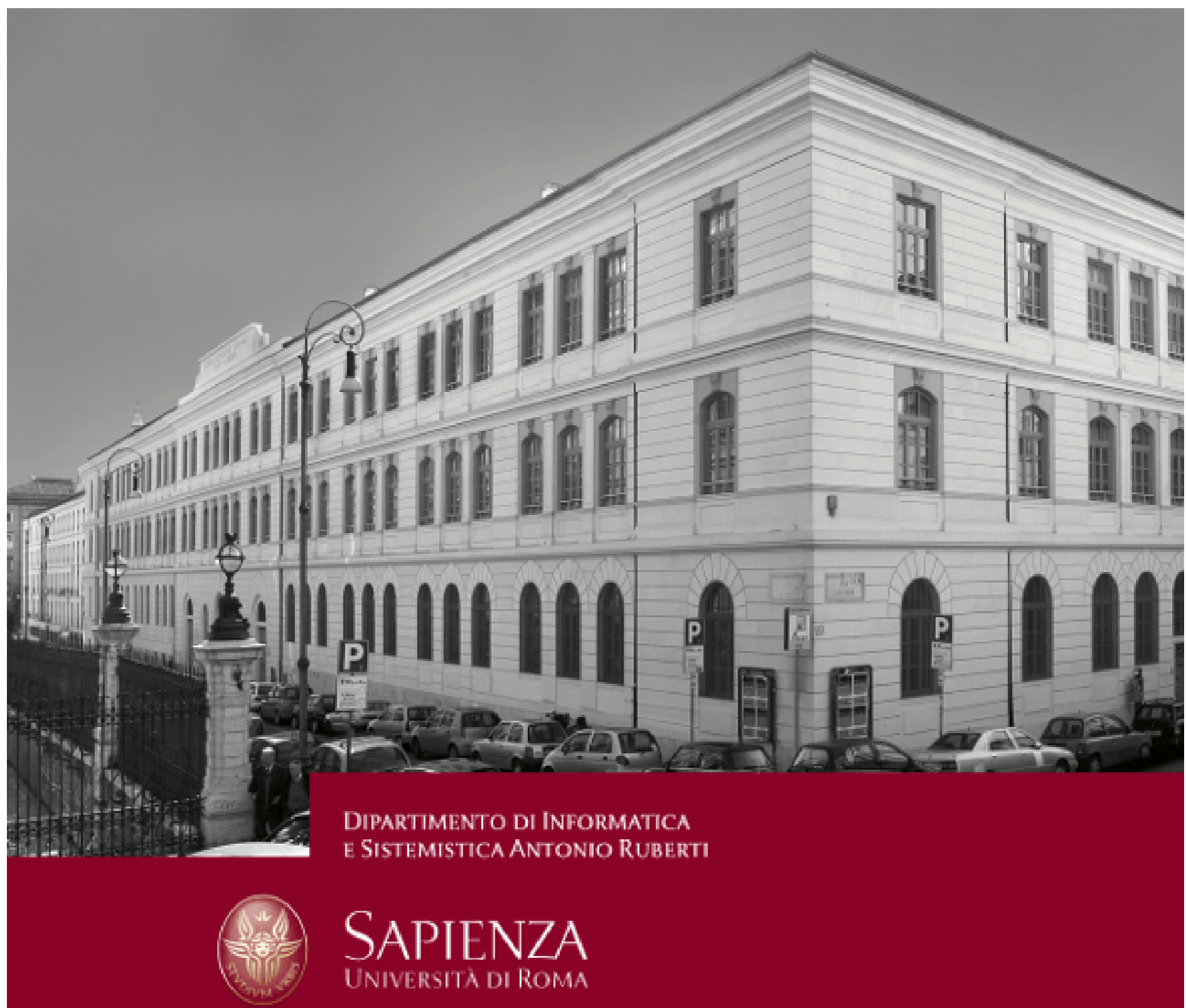

The Design of dynamical observers for hybrid systems: Theory and Application to an Automotive Control Problem

Andrea Balluchi

Luca Benvenuti

Maria D. Di Benedetto

Alberto L. Sangiovanni-Vincentelli

Technical Report n. 1, 2012 


\title{
The Design of dynamical observers for hybrid systems: Theory and Application to an Automotive Control Problem
}

\author{
Andrea Balluchi ${ }^{\dagger}$, Luca Benvenuti ${ }^{\S}$, Maria D. Di Benedetto ${ }^{b}$, \\ and Alberto L. Sangiovanni-Vincentelli ${ }^{\ddagger}$ \\ $\dagger$ Pure Power Control s.r.l, Via Giuntini 63, 56023 Navacchio di Cascina (PI), Italy \\ $\S$ DIS, "Sapienza" Università di Roma, Via Ariosto 25, 00185 Roma, Italy \\ b DIEL, Università dell'Aquila, Poggio di Roio, 67040 L'Aquila, Italy \\ $\ddagger$ EECS Dept., University of California at Berkeley, CA 94720, USA
}

Keywords: Hybrid systems observers, hybrid systems, discrete event systems, robust observers.

\begin{abstract}
A design methodology is presented for dynamical observers of hybrid systems with linear continuous-time dynamics that reconstruct the complete state (discrete location and continuous state) from the knowledge of the inputs and outputs of a hybrid plant. Given a current-location observable living hybrid system with minimum dwell-time, we prove that exponential ultimate boundedness for an hybrid observer can always be achieved. We also prove that the observer correctly identifies (apart from an initial finite number of transitions) the sequence of hybrid system locations even when the complementary outputs are generated with some delay with respect to the corresponding transitions in the plant.We then present the application of the theory to the problem of on-line identification of the actual engaged gear for a car, an important contribution. The relevance of this problem is related to engine control strategies achieving high performance and efficient emissions control which depend critically on the knowledge of the engaged gear. The performance of the observer was tested with (real and not simulated) experimental data obtained in Magneti Marelli Powertrain using an Opel Astra equipped with a Diesel engine and a robotized gearbox SeleSpeed.
\end{abstract}




\section{Introduction}

Hybrid systems are powerful abstractions for modeling complex systems. Their theoretical properties have been the subject of intense research. In addition, they have been used in a number of applications to provide models better reflecting the nature of control problems such as the ones related to embedded system design where discrete controls are routinely applied to continuous processes. Because of their generality, deriving rigorous controller synthesis procedures is often difficult. In many cases, we must resort either to heuristics or to approximations. Even when the structure of the hybrid problem is such that a controller can be synthesized, strong assumptions have to be used. For example, the use of hybrid formalisms to solve control problems in automotive applications (see $[7,6,4,5]$ ) has been proposed and control laws derived. These hybrid control algorithms are based on full state feedback. However, in most cases, only partial information about the state of the hybrid plant is available. Hence, a method for full state estimation is very important to make the hybrid control algorithms really applicable.

\section{$1.1 \quad$ Related work}

The state estimation problem had been the subject of intensive study for many years by both the computer science community in the discrete domain (see $[25,9,23]$ ), and the control community in the continuous domain (see the pioneering work of Kalman [15], Ackerson [1] and Luenberger [17]), but only scantly investigated in the hybrid system domain (e.g., see $[26,21,19,22,30,14]$ ).

More relevant to the problem we address is the literature on observer design. A complete theory and a design methodology for observers for mixed logical dynamical systems (MLD) was presented in [8] (see also [11]). This approach is applicable to any hybrid system that can be approximated by an MLD system and, consequently, it is a general powerful approach. However, even if a hybrid system could be well approximated by an MLD system, when "the observability horizon becomes large, solving the optimization problem can become computationally intractable" ([8]). In [12], algebraic necessary and sufficient conditions for the distinguishability of a linear switching system are offered. The authors propose a numerically efficient procedure for reconstruction of the state of the system as well as of switching signals and characterize the "bad" inputs to be avoided for which the continuous dynamics are indistinguishable. In [24], switched linear systems without reset and disturbances are considered. The author gives a condition for the existence of an observer based on the existence of a Lyapunov function that is common to the components of the system. In [27], observability for the same class of systems is first geometrically characterized. An observer is synthesized that generates the state estimate that converges to the actual state under persistent switching.

\subsection{Contributions of this paper}

We present a design methodology for dynamical observers of hybrid systems with linear continuoustime dynamics that reconstructs the complete state (discrete location and continuous state) from the knowledge of the inputs and outputs of a hybrid plant. The hybrid systems considered here have reset states and disturbances and hence, are more general than the ones addressed in [24, 27]. The proposed hybrid observer consists of two parts: a location observer that identifies the current 
location of the hybrid plant, and a continuous observer that produces an estimate of the evolution of the continuous state of the hybrid plant.

We first introduce the notion of current-location observable hybrid systems, for which the sequence of location switching can be identified by the location observer from the observation of the discrete plant output, without the need of additional information from the evolution of the continuous part of the plant. For this class of hybrid plants, the continuous observer can be designed by exploiting the results on stability of switching systems given in [20] and [13]. The proposed hybrid observer achieves correct identification of the location sequence and exponential convergence to zero of continuous observation error, or exponential ultimate boundedness in the case of noise.

We then extend the previous result to the case of not current-location observable hybrid systems. This can be achieved by using some additional information obtained from the processing of the continuous plant inputs and outputs for the identification of the sequence of location switchings. To do so, a set of additional discrete signals, called signatures, which are obtained from the continuous evolution of the system, is used in the location observer. We introduce the notion of hybrid systems that are current-location observable via signatures and propose a hybrid observer that achieves correct identification of the location sequence and exponential ultimate boundedness of continuous observation error.

The main contributions of this paper are the following:

- The proposed design methodology allows addressing observer synthesis for hybrid systems with partial discrete information on the current location, spanning the gap between the case of complete knowledge of current location (i.e. the hybrid plant produces as discrete output its current location), treated for instance in [3], and the case of absence of any discrete output information (i.e. the hybrid plant produces no discrete output), considered in $[8,11]$. This is an important result for applications where, typically, the discrete actions of the controller are known while no information regarding autonomous switching in the plant is available. Even if there is no discrete output (as in the industrial case we present in this paper), we do leverage the knowledge of the plant structure, i.e., the knowledge of the automaton associated to the hybrid system. Indeed, when we are in a particular location, we only take into consideration the signatures of the locations that are reachable in one step from the present one.

- When the discrete output information is not sufficient to identify the hybrid system location, i.e. the plant is not current-location observable, the processing of the continuous plant input/output signals in the signature generators is independent from the continuous state observation process. This is new with respect to previous approaches (see e.g. [14] and [21]) where the estimated values of continuous state of the plant are used to supply the missing information for the identification of the plant location. Our approach allows for separate tuning of the signature generators and the continuous observer. This aspect is quite appealing since the signature observer has to provide discrete information with fast transients regardless possible overshoots, while the continuous observer has to produce a smooth estimate of the continuous state with low sensitivity to noise.

- The complexity of the problem is mitigated by decoupling as much as possible the identification of the discrete location and the estimations of the continuous state. In particular, for 
current-location observable hybrid systems, the location sequence is identified independently from the continuous plant input/output evolution. Indeed, the location observer is a discrete event system and the continuous observer is a switching observer. In the general case, the location observer depends on the continuous plant input/output evolution but it includes only the minimum number of strictly necessary signature generators.

- Exponential stabilization of the dynamics of the continuous observation error is obtained by extending the results of stabilization by Morse [20] and Hespanha and Morse [13] to the class of switching systems with dwell-time and resets, subject to bounded disturbances.

- The proposed hybrid-observer design methodology was applied to an automotive control problem. We considered the problem of on-line identification of the actual engaged gear for a car. The relevance of this problem is related to engine control strategies achieving high performance and efficient emissions control which depend critically on the knowledge of the engaged gear. The performance of the observer was tested with (real and not simulated) experimental data obtained in Magneti Marelli Powertrain using an Opel Astra equipped with a Diesel engine and a robotized gearbox SeleSpeed. The specification given by Magneti Marelli Powertrain Division (a tier 1 automotive supplier) was to achieve correct identification on a set of maneuvers within a delay of $250 \mathrm{msec}$, using an implementation of the algorithm in discrete-time with a sampling period of $12 \mathrm{msec}$. The measurements were affected by delays, but the algorithm proved to be robust with respect to this non ideal situation. For the validation of the identification algorithm, the estimated engaged gear was compared to the signal on actual engaged gear provided by the control unit of the robotized gearbox.

\section{Problem formulation}

A hybrid system $\mathcal{H}$ is a collection

$$
\mathcal{H}=(Q, \Sigma, \Gamma, X, U, W, Y, \text { Init, } f, h, r, \delta, \zeta, \phi),
$$

where

- $Q, \Sigma$ and $\Gamma$ are the finite sets of discrete state, input and output variables, respectively;

- $X \subseteq \mathbb{R}^{n}, U \subseteq \mathbb{R}^{p}, W \subseteq \mathbb{R}^{n}$, and $Y \subseteq \mathbb{R}^{p}$ are the domains of continuous state, input, state disturbance and output variables, respectively;

- Init $\subseteq Q \times X$ is the set of admissible initial states;

- $f: Q \times X \times U \times W \rightarrow T X$ and $h: Q \times X \rightarrow Y$ are the vector fields defining the dynamics of the continuous state and output variables;

- $r: Q \times Q \times X \rightarrow X$ describes the continuous state resets.

- $\delta: Q \times \Sigma \rightarrow 2^{Q}$ and $\zeta: Q \times \Sigma \times Q \rightarrow \Gamma \bigcup\{\epsilon\}$ are, respectively, the set-valued functions defining the dynamics of the discrete state and output variables with $\epsilon$ being the null event; 
- $\phi: Q \times X \rightarrow 2^{\Sigma \bigcup\{\epsilon\}}$ is the set-valued function specifying the admissible events at each location $q \in Q$, for given values of the continuous state $x(t) \in X$.

The finite set $\Sigma$ of discrete inputs is composed by both internal events, auto-generated by the hybrid system on the basis of the values of the continuous state $x(t)$, and exogenous input events, whose enabling condition may or may not depend on $x(t)$. The null event $\epsilon$ is introduced to model different possible transition conditions. For example, if $\phi(q, x)=\{\epsilon\}$, then no input event is enabled for the given value of $x$ while if $\phi(q, x)=\{\sigma, \epsilon\}$, then the input event $\sigma$ is enabled. Moreover, if $\phi(q, x)=\{\sigma\}$, then the input event $\sigma$ is forced to occur and this can be used to model internal events forced to occur and auto-generated when the continuous state hits the boundary of the invariant associated to the discrete state.

In this paper, we consider living hybrid systems defined as those which admit only executions that are non-Zeno and have an infinite number of transitions (see [29]). For example, viable hybrid systems with 0-lag nonblocking control strategies and initial set Init equal to the viability kernel, are living hybrid systems (see [10]). Moreover, nonblocking hybrid systems with minimum and maximum dwell-time (see [26]) are also living hybrid systems.

For this class of hybrid systems a hybrid time basis $\tau=\left\{I_{k}\right\}_{k=0}^{\infty}$ is an infinite sequence of intervals, such that for all $k \geq 0, I_{k}=\left[t_{k}, t_{k}^{\prime}\right]$ with $t_{k} \leq t_{k}^{\prime}=t_{k+1}$.

Times $t_{k}$ denotes the times at which discrete transitions take place. If $t_{k}=t_{k}^{\prime}=t_{k+1}$, a multiple transition takes place at the same time.

In the sequel we will consider hybrid systems with no multiple transitions, i.e. hybrid systems for which all the time bases are such that $t_{k}<t_{k}^{\prime}=t_{k+1}$ for all $k>0$.

An execution of a living hybrid system with no multiple transitions will involve continuous evolution as well as instantaneous transitions (discrete evolution). In particular:

- $\left(q\left(t_{0}\right), x\left(t_{0}\right)\right) \in$ Init;

- (continuous evolution) for all $k$, when $t_{k} \leq t<t_{k+1}$,

$$
\begin{aligned}
q(t) & =q\left(t_{k}\right)=q(k) \\
\dot{x}(t) & =f(q(k), x(t), u(t), w(t)) \\
\gamma(t) & =\epsilon \\
y(t) & =h(q(k), x(t))
\end{aligned}
$$

and $\epsilon \in \phi(q(k), x(t))$;

- (discrete evolution) for all $k$, when $t=t_{k+1}$,

$$
\begin{aligned}
q(k+1) & \in \delta(q(k), \sigma(k+1)) \\
x\left(t_{k+1}\right) & =r\left(q(k), q(k+1), x\left(t_{k+1}^{-}\right)\right) \\
\gamma(k+1) & \in \zeta(q(k), \sigma(k+1), q(k+1)) \\
y\left(t_{k+1}\right) & =h\left(q(k+1), x\left(t_{k+1}\right)\right)
\end{aligned}
$$

and $\sigma(k+1) \in \phi\left(q(k), x\left(t_{k+1}^{-}\right)\right), \sigma(k+1) \neq \epsilon$ 


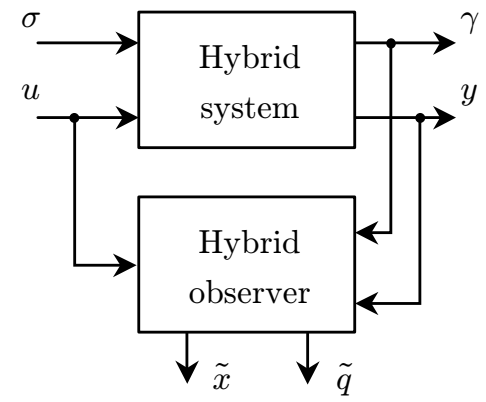

Figure 1: An observer for a hybrid system.

The output null event $\epsilon$ corresponds to an unobservable output. When a discrete output $\gamma \neq \epsilon$ is produced the corresponding transition is said to be observable.

In this paper, we consider the problem of designing a hybrid observer for living hybrid systems with no multiple transitions, see Figure 1.

Definition 1 A hybrid observer $\mathcal{O}$ of a given hybrid system $\mathcal{H}$ is a hybrid system that

- receives, as discrete input, the discrete output $\gamma(k)$ of $\mathcal{H}$ and, as continuous inputs, the continuous input $u(t)$ and output $y(t)$ of $\mathcal{H}$;

- provides as outputs estimates $\tilde{q}(k) \subseteq 2^{Q}$ and $\tilde{x}(t) \in \mathbb{R}^{n}$ of the current location $q(k)$ and continuous state $x(t)$ of the hybrid system $\mathcal{H}$, respectively.

We investigate the design of observers satisfying the following stability property:

Definition 2 Given a living hybrid systems with no multiple transitions $\mathcal{H}$ as in (2-9), a hybrid observer $\mathcal{O}$ is said to be exponentially ultimately bounded if there exists a positive integer $K$ and constants $c \geq 1, \mu>0$ and $b \geq 0$ for which

$$
\begin{aligned}
\tilde{q}(k) & =q(k) \quad \forall k \geq K \\
\|\tilde{x}(t)-x(t)\| & \leq c\left\|\tilde{x}\left(t_{K}\right)-x\left(t_{K}\right)\right\| e^{-\mu t}+b \quad \forall t>t_{K} .
\end{aligned}
$$

for every initial hybrid state $\left(q\left(t_{0}\right), x\left(t_{0}\right)\right) \in$ Init and every possible execution of $\mathcal{H}$. In (11), $\mu$ is the rate of convergence and $b$ is the ultimate bound. If $b=0$, the observer is said to be exponentially convergent.

In the sequel, we will assume that:

1. The functions $f$ and $h$ defining the dynamics of the continuous variables of the hybrid systems are linear and time-invariant:

$$
\begin{aligned}
f(q(k), x(t), u(t), w(t)) & =A_{k} x(t)+B_{k} u(t)+w(t) \\
h(q(k), x(t)) & =C_{k} x(t)
\end{aligned}
$$


where $w(t) \in \Omega$ is a bounded unknown disturbance such that

$$
\|w(t)\|_{\infty}=\max _{i=1, \ldots, n} \sup _{t \geq 0}\left|w_{i}(t)\right| \leq W,
$$

and the matrices $A_{k} \in \mathbb{R}^{n \times n}, B_{k} \in \mathbb{R}^{n \times m}, C_{k} \in \mathbb{R}^{p \times n}$ depend on the current system location $q(k)$.

2. The function $r$ describing the continuous state resets associated to the hybrid system transitions is affine:

$$
r\left(q_{i}, q_{j}, x\left(t_{k+1}^{-}\right)\right)=R_{i j}^{1} x\left(t_{k+1}^{-}\right)+R_{i j}^{0}
$$

where $t_{k+1}$ denotes the transition time from location $q(k)=q_{i}$ to location $q(k+1)=q_{j}$ and $R_{i j}^{1} \in \mathbb{R}^{n \times n}, R_{i j}^{0} \in \mathbb{R}^{n}$.

\section{Observers for current-location observable hybrid systems}

In this section we define a subclass of hybrid systems for which the design of exponentially ultimately bounded observers is made easy by the fact that the current location of the hybrid system can be identified independently from the system continuous evolution. This class of hybrid systems will be referred to as the class of current-location observable hybrid systems and a methodology for exponentially ultimately bounded observers design will be provided at the end of the section.

The proposed observer is a hybrid system itself. The methodology for the design of the observer is composed by two parts:

- The location observer design, which gives a DES that identifies, after a finite number of steps, the current location of the hybrid system using the discrete output information only;

- The continuous observer design, which associates to each location of the DES a continuous dynamics and to each transition a reset map, in such a way that the continuous evolution of the resulting hybrid system converges exponentially to that of the hybrid system.

\subsection{Current-location observable hybrid systems}

In this section, we define the class of current-location observable hybrid systems.

First, let us associate to a given hybrid system $\mathcal{H}$ as in (1) the nondeterministic DES

$$
\mathcal{D}_{\mathcal{H}}=(Q, \Sigma, \Gamma, \delta, \varphi, \zeta)
$$

with $\varphi$ defined as

$$
\varphi(q)=\bigcup_{x \in X} \phi(q, x) .
$$

The DES $\mathcal{D}_{\mathcal{H}}$ abstracts the dependence of the discrete dynamics of the hybrid system $\mathcal{H}$ from its continuous evolution.

Since the hybrid system $\mathcal{H}$ is a living hybrid system, then the DES $\mathcal{D}_{\mathcal{H}}$ is alive, that is a state at which no event is possible is never reached, formally $\forall q \in Q, \varphi(q) \neq \emptyset$. 
A state observer $\mathcal{D}_{\mathcal{O}}$ of the DES $\mathcal{D}_{\mathcal{H}}$ is a deterministic DES whose states are subsets of $2^{Q}$. At each time, the observer produces the subset of states the are feasible with the output sequence of $\mathcal{H}$ up to the current time. The observer is initialized in state $Q$. In order to construct such observer, let us define the set $Q_{\gamma}$ of states $q$ of $\mathcal{D}_{\mathcal{H}}$ such that there exists at least one transition to $q$ generating an observable output:

$$
Q_{\gamma}=\{q \in Q \mid \exists p \in Q, \sigma \in \varphi(p) \text {, such that } q \in \delta(p, \sigma) \text { and } \zeta(p, \sigma, q) \neq \epsilon\}
$$

By definition, when the DES $\mathcal{D}_{\mathcal{H}}$ produces an observable output $\gamma \neq \epsilon$, that is the observer receives an input $\gamma$, the state $q$ of the system must belong to the set $Q_{\gamma}$. Hence, apart from the initial state, the state space $\tilde{Q}$ of the observer is a subset of $2^{Q_{\gamma}}$.

Then, a straightforward design of an observer producing identification of the current state of $\mathcal{D}_{\mathcal{H}}$ after each observable output $\gamma$ can be obtained by computing the subset $\tilde{q} \in 2^{Q_{\gamma}}$ of possible states that the DES $\mathcal{D}_{\mathcal{H}}$ could have entered when the last output $\gamma$ was observed, taking into account the possible occurrence of one or more transitions giving $\epsilon$ as output prior to $\gamma$. This can be done in two steps:

1. Construct a DES $\mathcal{D}_{\mathcal{H}}^{\prime}$ having as inputs and outputs the observable outputs of $\mathcal{D}_{\mathcal{H}}$ and generating the same output language as $\mathcal{D}_{\mathcal{H}}$.

2. Construct the state observer $\mathcal{D}_{\mathcal{O}}$ for the DES $\mathcal{D}_{\mathcal{H}}{ }^{\prime}$ following the algorithm for the computation of the current-state observation tree, as described in ([]).

Let us now define a state of $\mathcal{D}_{\mathcal{H}}$ to be

- a persistent state if it may be visited after an arbitrarily long sequence of input events;

- an always-observable state if it can be identified every time the state is visited, except for a finite number of times in the beginning.

The set $P$ of all persistent states contains both states that are on a cycle and states that are in between cycles. In fact, altough the latter could be visited once, this visit may occur after an arbitrary long sequence of transitions.

An always-observable state has to be a singleton state in the observer and it should not be an element of any other persistent state of the observer which is not a singleton.

Let us now introduce the following definition:

Definition 3 The discrete event system $\mathcal{D}_{\mathcal{H}}$ is said to be current-state observable if there exists a positive integer $K$ such that for every $k \geq K$ and for any unknown initial state $q(0) \in Q$, the state $q(k)$ can be determined from the output sequence for every possible input and output sequence.

As shown by Ozveren and Willsky in [23], this notion of observability corresponds to requiring all persistent states of $\mathcal{D}_{\mathcal{H}}$ to be always-observable. Hence, the following theorem immediately follows

Theorem 4 The DES $\mathcal{D}_{\mathcal{H}}$ is current-location observable if and only if for every persistent state $q \in P$ of $\mathcal{D}_{\mathcal{H}}$ :

(C1) all exit transitions of $q$ are observable; 

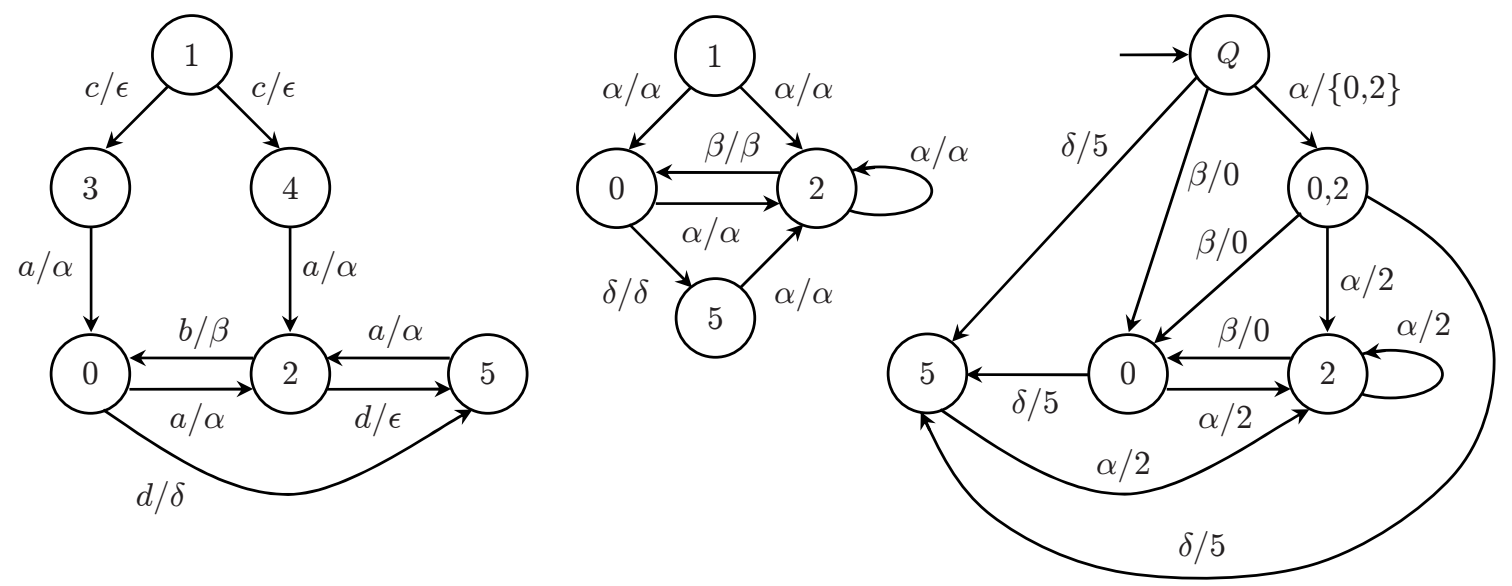

Figure 2: A DES $\mathcal{D}_{\mathcal{H}}$ (left), the DES $\mathcal{D}_{\mathcal{H}}^{\prime}$ (center) and the observer $\mathcal{D}_{\mathcal{O}}$ (right).
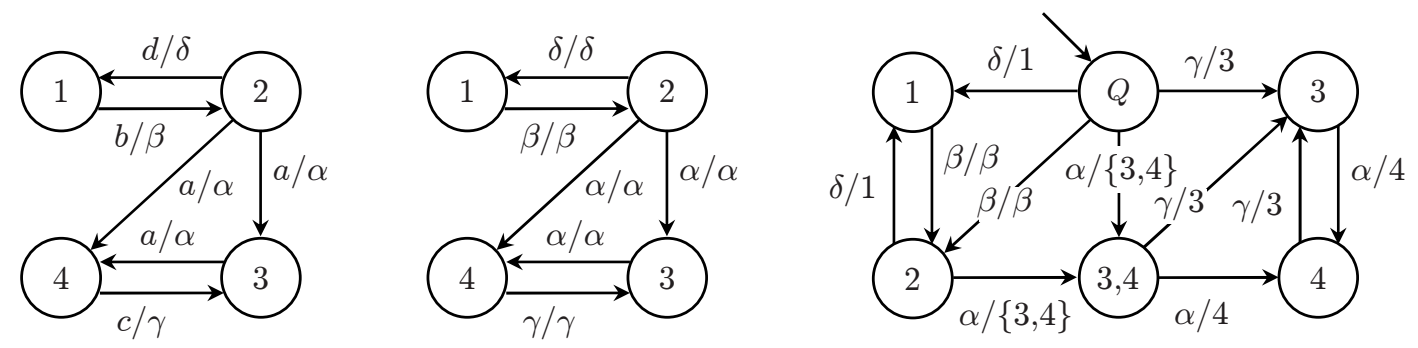

Figure 3: A DES $\mathcal{D}_{\mathcal{H}}$ (left), the $\operatorname{DES} \mathcal{D}_{\mathcal{H}}^{\prime}$ (center) and the observer $\mathcal{D}_{\mathcal{O}}$ (right).
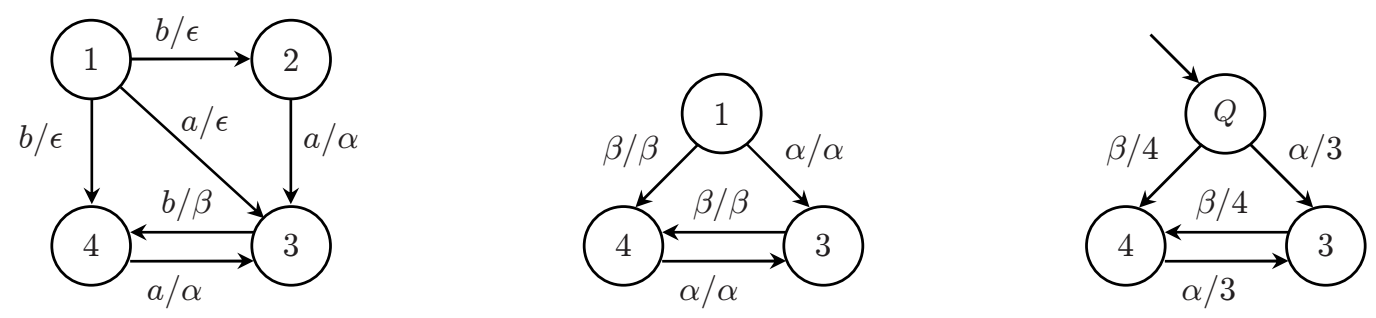

Figure 4: A DES $\mathcal{D}_{\mathcal{H}}$ (left), the DES $\mathcal{D}_{\mathcal{H}}^{\prime}$ (center) and the observer $\mathcal{D}_{\mathcal{O}}$ (right). 
(C2) there exists a singleton state $\{q\}$ in the observer $\mathcal{D}_{\mathcal{O}}$ and it is the only persistent state of $\mathcal{D}_{\mathcal{O}}$ containing $q$.

From the previos theorem it follows that $\mathcal{D}_{\mathcal{H}}$ and $\mathcal{D}_{\mathcal{O}}$ have the same set of persistent states.

As an example, consider the DES $\mathcal{D}_{\mathcal{H}}$ depicted on the left hand side of Figure 2. Pair $(\sigma / \gamma)$ associated to each arc denotes the input and output events. The corresponding DES $\mathcal{D}_{\mathcal{H}}^{\prime}$ and the observer $\mathcal{D}_{\mathcal{O}}$ are depicted in the middle and on the right hand side of Figure 2, respectively. The persistent states of $\mathcal{D}_{\mathcal{H}}$ are $P=\{0,2,5\}$. Since $\mathcal{D}_{\mathcal{O}}$ has the same set of persistent states, then condition (C2) holds. However, since there is a transition with unobservable output from 2 to 5 , then condition (C1) fails and the DES $\mathcal{D}_{\mathcal{H}}$ is not current-state observable. In fact, when $\mathcal{D}_{\mathcal{H}}$ is in state 2 and it receives the event $d$, the corresponding transition to 5 is not detected by the observer $\mathcal{D}_{\mathcal{O}}$.

It is worth noting that a DES $\mathcal{D}_{\mathcal{H}}$ can be not current-state observable even if all the transitions produce an observable output. As an example, consider the DES $\mathcal{D}_{\mathcal{H}}$ depicted on the left hand side of Figure 3. The corresponding DES $\mathcal{D}_{\mathcal{H}}^{\prime}$ and the observer $\mathcal{D}_{\mathcal{O}}$ are depicted in the middle and at the right hand side of Figure 3, respectively. All the states of $\mathcal{D}_{\mathcal{H}}$ are persistent states and condition (C1) holds. The set of persistent states of the observer $\mathcal{D}_{\mathcal{O}}$ is $\{\{1\},\{2\},\{3\},\{4\},\{3,4\}\}$. Since the state 3 and 4 appear in the observer persistent state $\{3,4\}$, which is not a singleton, then condition (C2) is not verified and the DES $\mathcal{D}_{\mathcal{H}}$ is not current-state observable. In fact, if the DES $\mathcal{D}_{\mathcal{H}}$ starts from location 1 or 2 , then it may visit locations 3 and 4 after an arbitrary long sequence of transitions between locations 1 and 2 . When this occurs, the observer $\mathcal{D}_{\mathcal{O}}$ is not able to recognize which of the two locations 3 and 4 is entered.

Finally, an example of a current-state observable DES $\mathcal{D}_{\mathcal{H}}$ is depicted on the left hand side of Figure 4. The corresponding DES $\mathcal{D}_{\mathcal{H}}^{\prime}$ and the observer $\mathcal{D}_{\mathcal{O}}$ are depicted in the middle and on the right hand side of Figure 4, respectively. It is readely verified that conditions (C1) and (C2) are fullfilled.

The notion of current-state observability for DESs induces a similar notion for hybrid systems as follows:

Definition 5 A living hybrid system with no multiple transitions $\mathcal{H}$ as in (2-9) is said to be current-location observable if the associated nondeterministic DES $\mathcal{D}_{\mathcal{H}}$ is current-state observable.

\subsection{Hybrid observer design}

Given a current-location observable living hybrid system with no multiple transitions $\mathcal{H}$ as in $(2-9)$, the proposed observer $\mathcal{H}_{\mathcal{O}}$ is a hybrid system itself defined as follows.

Location-observer design. The discrete behavior of the hybrid observer $\mathcal{H}_{\mathcal{O}}$ is defined by the observer $\mathcal{D}_{\mathcal{O}}$ for the DES $\mathcal{D}_{\mathcal{H}}$ associated to the hybrid system. For current-location observable hybrid systems, by Definitions 3 and 5 , there exists a finite $K$ such that $\tilde{q}(k)=q(k)$ for any $k \geq K$. The time $t_{K}$ from which the hybrid system location is properly identified corresponds to the time at which $\mathcal{D}_{\mathcal{O}}$ enters the set $P$ of persistent states. Moreover, for $t>t_{K}$, the transitions of the DES $\mathcal{D}_{\mathcal{O}}$ are synchronous with the hybrid system transitions. 
Continuous-observer design. The continuous behavior of the hybrid observer, which determines the evolution of the estimate $\tilde{x}(t)$ of the system continuous state $x(t)$, is defined as follows:

1. to each observer location $\tilde{q}$ is associated the continuous dynamics

$$
\dot{\tilde{x}}(t)=\tilde{f}(\tilde{q}, \tilde{x}(t), u(t), y(t))
$$

where

$$
\tilde{f}= \begin{cases}0 & \text { if } \tilde{q} \notin P \\ \left(A_{i}-G_{i} C_{i}\right) \tilde{x}(t)+B_{i} u(t)+G_{i} y(t) & \text { if } \tilde{q}=\left\{q_{i}\right\} \in P\end{cases}
$$

and $A_{i}, B_{i}, C_{i}$ are as in (12-13), and the observer gain matrix $G_{i} \in \mathbb{R}^{n \times p}$ is the design parameter used to set the velocity of convergence in each location $\tilde{q} \in P$.

The function $\tilde{f}$ is such that, for $t \leq t_{K}$, i.e. before the hybrid system location has been identified, the continuous observer state $\tilde{x}$ is locked at its initial value $\tilde{x}(0)$. Then, for $t \geq t_{K}$ the function $\tilde{f}$ implements a switching Luenberger's observer (see the survey work [17]). Since hybrid observer transitions and hybrid system transitions are synchronous, then the dynamic parameters of the observer switch synchronously with the hybrid system parameters.

2. to each observer transition $\left\{q_{i}\right\} \rightarrow\left\{q_{j}\right\}$, with $\left\{q_{i}\right\} \in P$ and $^{1} q_{j} \in \operatorname{Reach}\left(q_{i}\right)$, is associated the reset

$$
\tilde{x}\left(t_{k}\right)=\tilde{x}\left(t_{k}^{+}\right)=\tilde{r}\left(\left\{q_{i}\right\},\left\{q_{j}\right\}, \tilde{x}\left(t_{k}^{-}\right)\right)=R_{i j}^{1} \tilde{x}\left(t_{k}^{-}\right)+R_{i j}^{0}
$$

where $t_{k}$ denotes the observer transition time and $R_{i j}^{1}, R_{i j}^{0}$ are as in (15).

Due to the synchronization between hybrid observer and hybrid system transitions, the hybrid observer applies the same reset of the hybrid system at the same time.

The scheme of the proposed hybrid observer is depicted in figure 5

Exponential ultimate boundedness Introducing the observation error $\zeta=\tilde{x}-x$, by (16) and $(12-13)$, for $t \geq t_{K}$ we have

$$
\dot{\zeta}(t)=\left(A_{i}-G_{i} C_{i}\right) \zeta(t)-w(t)
$$

with, by (17) and (15), resets at system switching times $t_{k}$ equal to

$$
\zeta\left(t_{k}\right)=\zeta\left(t_{k}^{+}\right)=R_{i j}^{1} \zeta\left(t_{k}^{-}\right)
$$

As pointed out in [2], the stabilization of the hybrid dynamics of the observation error is more complex than the stabilization of each single dynamics in (16). Sufficient conditions for exponential convergence of the hybrid observer can be obtained for hybrid systems $\mathcal{H}$ that exhibit a minimum dwell time between location switching. Given a current-location observable living hybrid system with minimum dwell-time, the next theorem states that exponential ultimate boundedness can always be achieved:

\footnotetext{
${ }^{1} \operatorname{Reach}\left(q_{i}\right)$ denotes the set of locations reachable in one step from $q_{i}$, that is $\operatorname{Reach}\left(q_{i}\right)=$ $\left\{q \mid q \in \delta\left(q_{i}, \sigma\right)\right.$ with $\left.\sigma \in \varphi\left(q_{i}\right)\right\}$.
} 


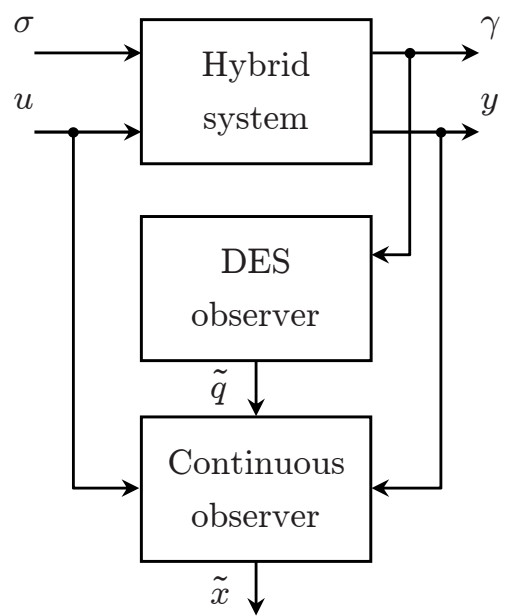

Figure 5: The scheme of the hybrid observer for a current-location observable hybrid system.

Theorem 6 Given a current-location observable living hybrid system $\mathcal{H}$, as in (2-9), with minimum dwell-time $D$ and such that all the pairs $\left(A_{i}, C_{i}\right)$ are observable for every persistent state $q_{i}$, then for any rate of convergence $\mu>0$, there exists a value for the minimum dwell-time $D$ such that the hybrid observer $\mathcal{H}_{O}$ is exponentially ultimately bounded with rate of convergence $\mu$ and with ultimate bound $b$ proportional to $W$ - exponentially convergent in the absence of disturbance.

The proof of the theorem above (see Appendix) is based on the fact that, if each $A_{i}-G_{i} C_{i}$ has distinct eigenvalues, then the rate of convergence of the continuous state observation error $\zeta=\tilde{x}-x$ is lower bounded by

$$
-\alpha\left(A_{i}-G_{i} C_{i}\right)-\frac{\max \left\{0, \log \left[r_{i}^{1} k\left(A_{i}-G_{i} C_{i}\right)\right]\right\}}{D},
$$

where $r_{i}^{1}=\max _{q_{j} \in \operatorname{Reach}\left(q_{i}\right)}\left\|R_{i j}^{1}\right\|$, and $\alpha(A)$ and $k(A)=\|T\|\left\|T^{-1}\right\|$ denote, respectively, the spectral abscissa and condition number of the matrix $A$, with respect to inversion of the matrix $T$ such that $T^{-1} A T$ is in the Jordan canonical form.

Hence, if

$$
\alpha\left(A_{i}-G_{i} C_{i}\right)+\frac{\max \left\{0, \log \left[r_{i}^{1} k\left(A_{i}-G_{i} C_{i}\right)\right]\right\}}{D} \leq-\mu<0
$$

then the rate of convergence $\mu$ is achieved. For any rate of convergence $\mu$ there always exist matrices $G_{i}$ such that $(20)$ is satisfied, provided that the pair $\left(A_{i}, C_{i}\right)$ is observable and $D$ is large enough.

The previous result is an extension of a popular result by Morse [13] on switching systems stability to the case of robust stability of hybrid systems with continuous state resets. 


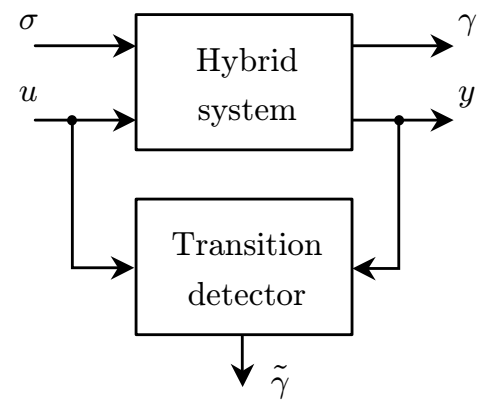

Figure 6: The Transition detector.

\section{Observers for generic hybrid systems}

\subsection{Recovering current-location observability: the transition detector}

The discrete state of a hybrid system $\mathcal{H}$ may be correctly identified even if $\mathcal{H}$ is not current-location observable. This may be achieved by processing the continuous inputs and outputs of $\mathcal{H}$ to obtain some additional information for discrete state identification. The system $\mathcal{T}_{\mathcal{H}}$ that is introduced to this purpose is called transition detector (see Figure 6). The transition detector processes the continuous input $u(t)$ and output $y(t)$ of the hybrid system $\mathcal{H}$ and generates some additional discrete signals $\tilde{\gamma} \in \tilde{\Gamma}$, referred to as complementary discrete outputs, to be used as extra inputs by the DES observer. More precisely, by Theorem 4, if the hybrid system is not current-location observable, then at least one of the conditions (C1) and (C2) fails. When condition (C1) fails, i.e. there exists one persistent state of $\mathcal{D}_{\mathcal{H}}$ having an unobservable exit transition, the transition detector has to detect this transition and produce a corresponding output event $s \in \tilde{\Gamma}$. When condition (C2) fails, i.e. there exists a persistent non-singleton state of $\mathcal{D}_{\mathcal{O}}$ containing more than one persistent state of $\mathcal{D}_{\mathcal{H}}$, the transition detector has to distinguish one transition from the others and produce a corresponding output event $s \in \tilde{\Gamma}$.

The DES obtained from the DES $\mathcal{D}_{\mathcal{H}}$ associated to $\mathcal{H}$ by enriching its outputs with the complementary discrete outputs generated by $\mathcal{T}_{\mathcal{H}}$, will be denoted by $\hat{\mathcal{D}}_{\mathcal{H}}$ and referred to as the complementary DES associated to the composition of the hybrid system $\mathcal{H}$ and the transition detector $\mathcal{T}_{\mathcal{H}}$.

Consider for example a hybrid system whose discrete behavior is represented by the DES $\mathcal{D}_{\mathcal{H}}$ shown in Figure 2, which is not current-location observable since condition (C1) fails. Assume that a complementary discrete output, referred to as $s_{5}$, is produced by the transition detector when it detects the unobservable transition from location 2 to location 5 .

The complementary DES $\hat{\mathcal{D}}_{\mathcal{H}}$, shown in Figure 7 , can be obtained by adding in the original DES $\mathcal{D}_{\mathcal{H}}$ the complementary output $s_{5}$ to the unobservable transition from location 2 to location 5. As it can be easily checked, the introduction of the complementary output makes the DES $\hat{\mathcal{D}}_{\mathcal{H}}$ current-state observable. The observer $\hat{\mathcal{D}}_{\mathcal{O}}$ obtained applying the synthesis described in Section 3 is shown in Figure 7.

In a similar way, consider a hybrid system whose discrete behavior is represented by the DES $\mathcal{D}_{\mathcal{H}}$ shown in Figure 3, which is not current-location observable since condition (C2) fails. Assume 

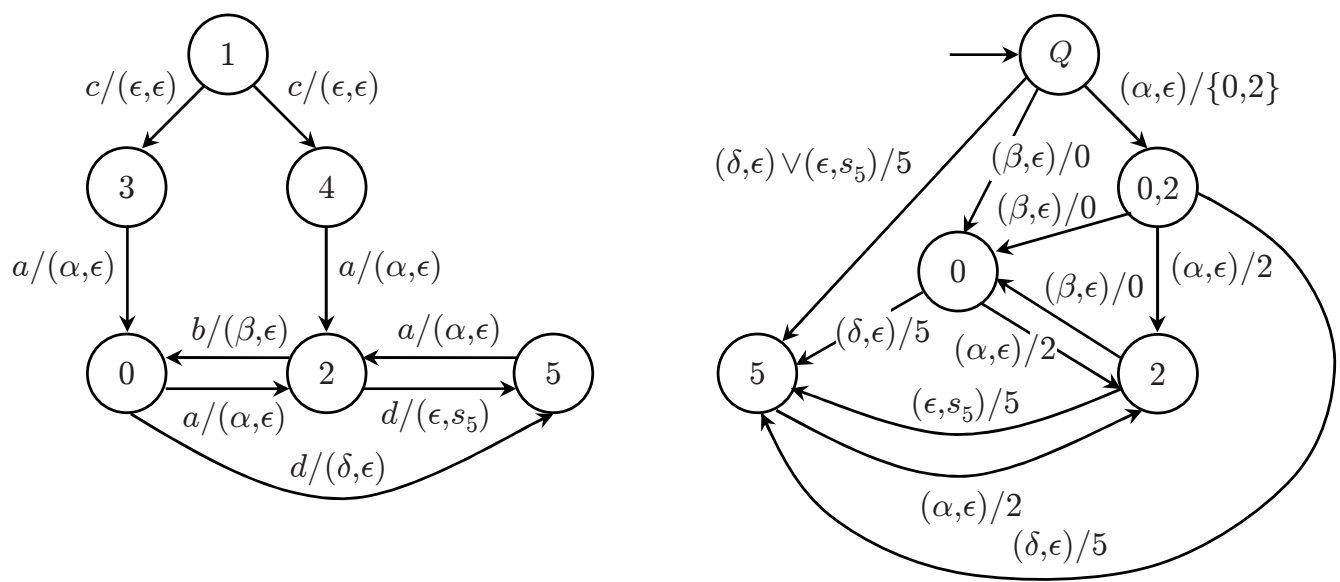

Figure 7: The DES $\hat{\mathcal{D}}_{\mathcal{H}}$ (left) and the observer $\hat{\mathcal{D}}_{\mathcal{O}}$ (right).
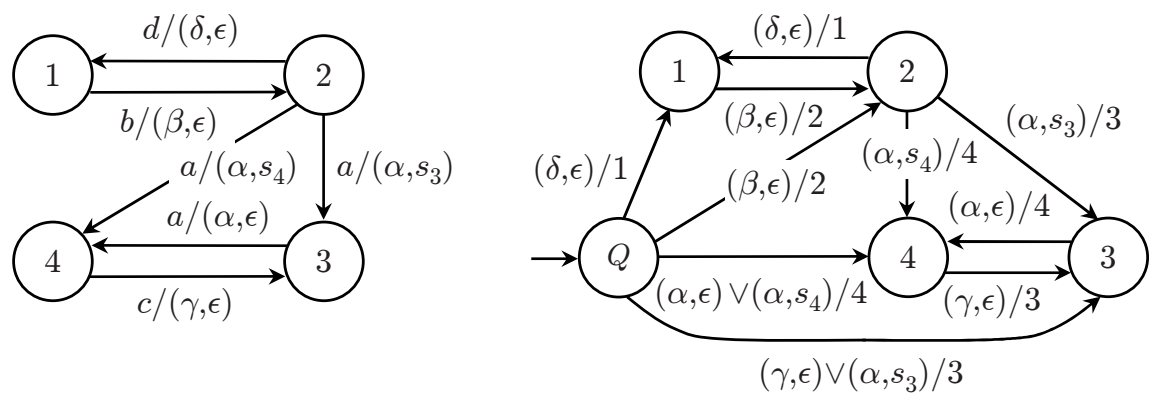

Figure 8: The DES $\hat{\mathcal{D}}_{\mathcal{H}}$ (left), the DES $\hat{\mathcal{D}}_{\mathcal{H}}^{\prime}$ (center) and the observer $\hat{\mathcal{D}}_{\mathcal{O}}$ (right).

that two complementary discrete outputs, referred to as $s_{3}$ and $s_{4}$, are produced by the transition detector when the transition from location 2 to location 3 and that from location 2 to location 4 are detected, respectively. The complementary DES $\hat{\mathcal{D}}_{\mathcal{H}}$,shown in Figure 8, can be obtained by adding in the original DES $\mathcal{D}_{\mathcal{H}}$ the complementary outputs $s_{3}$ and $s_{4}$ to the transitions from location 2 to locations 3 and 4 , respectively.

As it can be easily checked, the introduction of the complementary outputs makes the DES $\hat{\mathcal{D}}_{\mathcal{H}}$ current-state observable. The observer $\hat{\mathcal{D}}_{\mathcal{O}}$ is shown in Figure 8.

In Figure 6, the composition of a given not current-location observable hybrid system $\mathcal{H}$ with the transition detector is depicted. If the transition detector $\mathcal{T}_{\mathcal{H}}$ is such that the complementary DES $\hat{\mathcal{D}}_{\mathcal{H}}$ associated to the composition of $\mathcal{H}$ and $\mathcal{T}_{\mathcal{H}}$ is current-state observable, then the hybrid system is said to be current-location recoverable and the transition detector is referred to as an admissible one.

We do not discuss in this paper general mehods for the construction of a transition detector $\mathcal{T}_{\mathcal{H}}$ that, for example, can be based on failure detection and identification techniques (see [18] for a tutorial). However, in Section 5 we show how a transition detector $\mathcal{T}_{\mathcal{H}}$ can be successfully determined for the the design of a location-observer in an automotive application. 
Consider then a current-location recoverable hybrid system $\mathcal{H}$ and an admissible transition detector $\mathcal{T}_{\mathcal{H}}$ for $\mathcal{H}$. From the previous definition it follows that if $\mathcal{T}_{\mathcal{H}}$ generates the complementary discrete outputs $\tilde{\gamma}$ synchronously with the corresponding hybrid system transitions, then the observer $\hat{\mathcal{D}}_{\mathcal{O}}$ is able to identify the current-location of the hybrid system $\mathcal{H}$. Moreover, the observer $\hat{\mathcal{D}}_{\mathcal{O}}$ correctly identifies (apart from an initial finite number of transitions) the sequence of hybrid system locations even when the complementary outputs are generated with some delay with respect to the corresponding transitions in $\mathcal{H}$ provided that $\mathcal{T}_{\mathcal{H}}$ is able to detect any transition of $\mathcal{H}$ for which a complementary output has to be produced, before the subsequent transition of $\mathcal{H}$ takes place.

\subsection{Hybrid observer design}

Given a current-location recoverable hybrid system with no multiple transitions $\mathcal{H}$ as in $(2-9)$ assume that

(C) there exists an admissible transition detector $\mathcal{T}_{\mathcal{H}}$ able to detect any transition of $\mathcal{H}$ for which a complementary output has to be produced, before the subsequent transition of $\mathcal{H}$ takes place.

Then the proposed observer $\mathcal{H}_{\mathcal{O}}$ is a hybrid system itself defined as follows.

Location-observer design. The discrete behavior of the hybrid observer $\mathcal{H}_{\mathcal{O}}$ is defined by the observer $\hat{\mathcal{D}}_{\mathcal{O}}$ for the DES $\hat{\mathcal{D}}_{\mathcal{H}}$ associated to the composition of $\mathcal{H}$ and $\mathcal{T}_{\mathcal{H}}$. For current-location recoverable hybrid systems, if the transition detector satisfies condition $(\mathbf{C})$, then there exists a finite $K$ such that $\tilde{q}(k)=q(k)$ for any $k \geq K$. The time $t_{K}$ from which the hybrid system location sequence is properly identified corresponds to the time at which $\hat{\mathcal{D}}_{\mathcal{H}}$ enters the set $P$ of persistent states. For $t>t_{K}$, the transitions of the DES $\hat{\mathcal{D}}_{\mathcal{H}}$ are, in general, not synchronous with the hybrid system transitions. More in detail, the transition of the DES $\hat{\mathcal{D}}_{\mathcal{H}}$ associated to a complementary output null event are synchronous with the corresponding hybrid system transition, while those associated to a non null complementary output may take place with a delay with respect to the corresponding hybrid system transition. Nonetheless, for $t>t_{K}$, the observer $\hat{\mathcal{D}}_{\mathcal{O}}$ correctly identifies the sequence of hybrid system location. In fact, let us denote by $t_{k}^{\prime}$ the time at which the transition detector generates the complementary output associated to the $k$-th hybrid system transition. Since, by assumption, the transition detector satisfies condition (C), then

$$
t_{k} \leq t_{k}^{\prime}<t_{k+1}
$$

where $t_{k}$ is the time at which the $k$-th hybrid system transition takes place. Hence, for $t>t_{K}$

$$
\tilde{q}(t)=q\left(t_{k}\right)=q(k) \quad \text { for } t_{k}^{\prime} \leq t<t_{k+1}^{\prime}
$$

As an example, consider a not current-location observable hybrid system $\mathcal{H}$ whose discrete behavior is represented by the DES $\mathcal{D}_{\mathcal{H}}$ shown in Figure 2 and consider the admissible transition detector $\mathcal{T}_{\mathcal{H}}$ generating the complementary discrete output $s_{5}$ when the unobservable transition from location 2 to location 5 is detected (see Figure 7). Assume that the complementary output $s_{5}$ is generated 


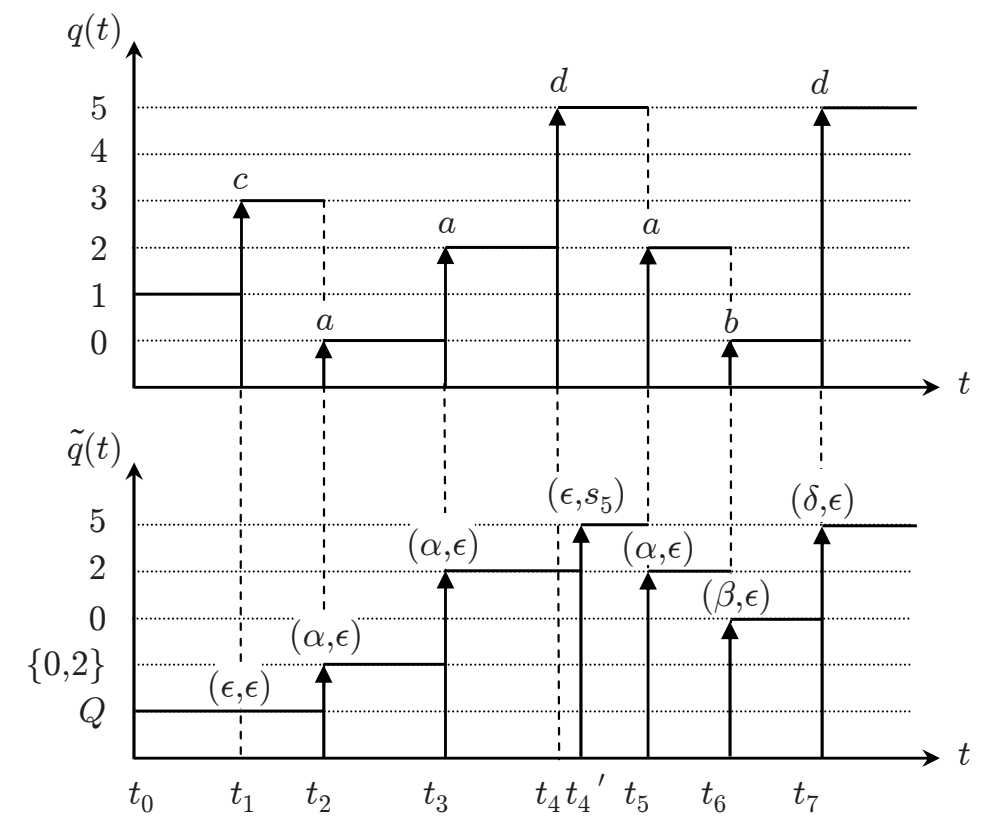

Figure 9: A possible discrete evolution of the hybrid system considered in this Section (upper figure) and the corresponding evolution of the observer location (lower figure).

with a delay with respect to the unobservable hybrid system transition from location 2 to location 5 but always before the next transition of the hybrid system.

A possible discrete evolution of the hybrid system starting from location 1 and corresponding to the discrete input sequence $\{c, a, a, d, a, b, d, \ldots\}$ is depicted in the upper picture of Figure 9. The location sequence $q(k)$ is $\{1,3,0,2,5,2,0,5, \ldots\}$ and the discrete output sequence is $\{\epsilon, \alpha, \alpha, \epsilon, \alpha, \beta, \delta, \ldots\}$. The input sequence to the observer $\hat{\mathcal{D}}_{\mathcal{O}}$ (see Figure 7 ) is then

$$
\left\{(\epsilon, \epsilon),(\alpha, \epsilon),(\alpha, \epsilon),\left(\epsilon, s_{5}\right),(\alpha, \epsilon),(\beta, \epsilon),(\delta, \epsilon), \ldots\right\}
$$

The evolution of the observer location, that is $\tilde{q}(k)$, is depicted in the lower picture of Figure 9 . The observer location sequence is $\{Q,\{0,2\}, 2,5,2,0,5, \ldots\}$. As the figure makes clear, form the time $t_{K}=t_{3}$ the hybrid system location sequence is properly identified even if there is a delay $t_{4}^{\prime}-t_{4}$ in detecting the transition from location 2 to location 5.

The scheme of the proposed hybrid observer is depicted in figure 10.

Continuous-observer design. The continuous behavior of the hybrid observer, which determines the evolution of the estimate $\tilde{x}(t)$ of the system continuous state $x(t)$, is defined as follows:

1. to each observer location $\tilde{q}$ is associated the continuous dynamics

$$
\dot{\tilde{x}}(t)=\tilde{f}(\tilde{q}, \tilde{x}(t), u(t), y(t))
$$




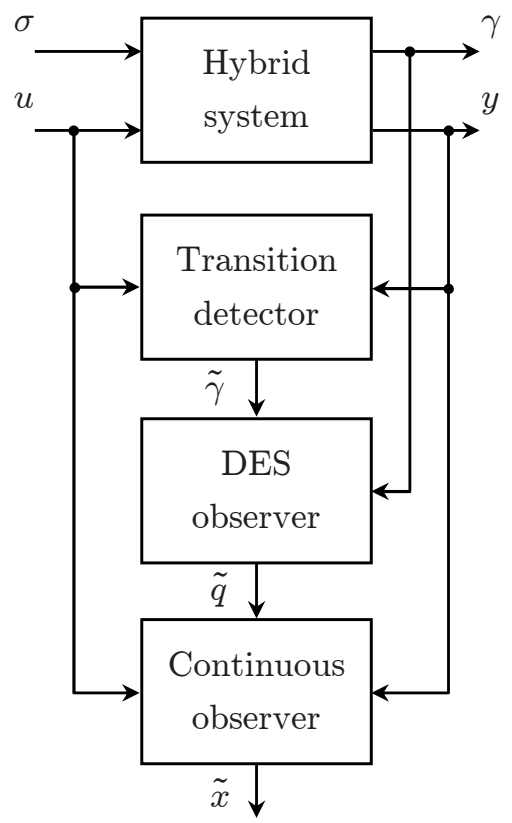

Figure 10: The scheme of the hybrid observer for a current-location recoverable hybrid system.

where

$$
\tilde{f}= \begin{cases}0 & \text { if } \tilde{q} \notin P \\ \left(A_{i}-G_{i} C_{i}\right) \tilde{x}(t)+B_{i} u(t)+G_{i} y(t) & \text { if } \tilde{q}=\left\{q_{i}\right\} \in P\end{cases}
$$

and $A_{i}, B_{i}, C_{i}$ are as in (12-13), and the observer gain matrix $G_{i} \in \mathbb{R}^{n \times p}$ is the design parameter used to set the velocity of convergence in each location $\tilde{q} \in P$.

The function $\tilde{f}$ is such that, for $t \leq t_{K}$, i.e. before the hybrid system location has been identified, the continuous observer state $\tilde{x}$ is locked at its initial value $\tilde{x}(0)$. Then, for $t \geq t_{K}$ the function $\tilde{f}$ implements a switching Luenberger's observer. In this case, since hybrid observer transitions and hybrid system transitions may be not synchronous, then the dynamic parameters of the observer may switch with a delay with respect to the hybrid system parameters.

2. to each observer transition $\left\{q_{i}\right\} \rightarrow\left\{q_{j}\right\}$, with $\left\{q_{i}\right\} \in P$ and $q_{j} \in \operatorname{Reach}\left(q_{i}\right)$, is associated the reset

$$
\tilde{x}\left(t_{k}^{\prime}\right)=\tilde{x}\left(t_{k}^{\prime+}\right)=\tilde{r}\left(\left\{q_{i}\right\},\left\{q_{j}\right\}, \tilde{x}\left(t_{k}^{\prime-}\right)\right)=R_{i j}^{1} \tilde{x}\left(t_{k}^{\prime-}\right)+R_{i j}^{0}
$$

where $t_{k}^{\prime}$ denotes the observer transition time and $R_{i j}^{1}, R_{i j}^{0}$ are as in (15).

Due to the possible delay between hybrid observer and hybrid system transitions, the hybrid observer may apply the same reset of the hybrid system with a delay.

Exponential ultimate boundedness As in Section 3, sufficient conditions for exponential convergence of the hybrid observer can be obtained for hybrid systems $\mathcal{H}$ that exhibit a minimum dwell time between location switching: 
Theorem 7 Given a current-location recoverable living hybrid system $\mathcal{H}$, as in (2-9), with minimum dwell time $D$ and such that all the pairs $\left(A_{i}, C_{i}\right)$ are observable for every persistent state $q_{i}$, and a transition detector $\mathcal{T}_{\mathcal{H}}$ able to detect within a time $\Delta<D$ any transition of $\mathcal{H}$ for which a complementary output has to be produced, then for any rate of convergence $\mu>0$, there exists a value for the minimum dwell-time $D$ such that the hybrid observer $\mathcal{H}_{O}$ is exponentially ultimately bounded with rate of convergence $\mu$.

The proof of the above theorem can be found in Appendix.

\section{An application to automotive control}

In this section we present some experimental results describing the application of the proposed hybrid-observer design methodology to an automotive control problem. In more detail, we consider the problem of on-line identification of the actual engaged gear for a car. The relevance of this problem is related to engine control strategies achieving high performance and efficient emissions control which depend critically on the knowledge of the engaged gear. In fact, this information is necessary in engine torque control to compensate the equivalent inertia of the vehicle on the crankshaft. Moreover, for Diesel engines, actual gear identification is very important to improve emissions control since particulate emissions (which consist of soot with some additional absorbed hydrocarbon materials) are particularly difficult to control with first gear engaged. At present, in commercial cars, the problem of actual engaged gear identification is solved by comparing the revolution speed of the wheels with the revolution speed of the crankshaft. However, since both of them are affected by oscillations due to the elasticity of the transmission shafts and the tires, then this approach implies large time delays in the identification and may produce significant identification errors.

The problem is here solved by considering a hybrid model of the driveline in which the engaged gear and connection clutch state are represented as discrete states. As a consequence, the gear identification problem reduces to the identification of the current location of the driveline hybrid model.

The model of the driveline here considered is represented as two main inertias (the crankshaft and the body vehicle) connected by an elasticity, a gear and a clutch, as depicted in in Figure 11. Since the gear can have different ratios and the clucth can be in different operating modes (locked, open or slipping), then the behavior of the driveline is described by a hybrid model $\mathcal{H}$ where the engaged gear and connection clutch state are just represented as discrete states. In more detail, the model $\mathcal{H}$ has 7 locations, i.e.

$$
q \in\left\{q_{1}, q_{2}, q_{3}, q_{4}, q_{5}, q_{R}, q_{N}\right\}
$$

where locations $q_{i}$, for $i=1, \ldots, 5$, correspond to $i$-th gear engaged and clutch locked, location $q_{R}$ models reverse gear engaged and clutch locked, and location $q_{N}$ represents either driveline open (idle gear and/or clutch open) or clutch slipping. The DES $\mathcal{D}_{\mathcal{H}}$ describing the discrete dynamics of $\mathcal{H}$ is depicted in Figure 12.

The inputs of the hybrid model are both discrete and continuous. The position of the gear lever lever $\in\{1,2,3,4,5, R, N\}$ is the discrete input while the connection pressure of the clutch 


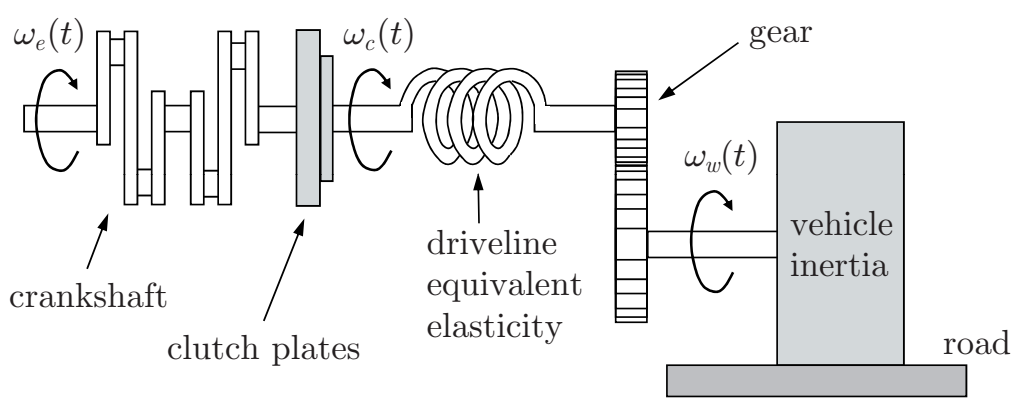

Figure 11: Scheme of the driveline model

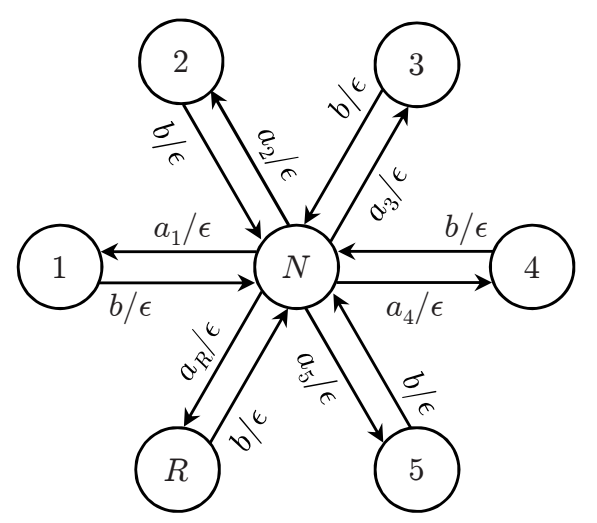

Figure 12: The DES $\mathcal{D}_{\mathcal{H}}$ associated to the driveline hybrid model $\mathcal{H}$. 
plates $P_{c}(t)$ and the torque generated by the engine $T_{e}(t)$ are the continuous inputs. Moreover, the torque acting on the wheels $T_{w}(t)$ is unknown and has to be considered as a continuous time input disturbance. The continuous state variables are the driveline torsion angle $\alpha(t)$, the crankshaft revolution speed $\omega_{e}(t)$, the clutch revolution speed $\omega_{c}(t)$ and the wheel revolution speed $\omega_{w}(t)$.

When the clutch is locked then $\omega_{e}(t)=\omega_{c}(t)$ and the crankshaft transmits to the driveline the torque

$$
T_{c}(t)=k_{i} \alpha(t)+b_{i}\left(\omega_{e}(t)-\frac{\omega_{w}(t)}{\tau_{i}}\right)
$$

where $\tau_{i}$ is the transmission ratio, and $k_{i}$ and $b_{i}$ are the driveline equivalent elasticity and damping coefficients for $i$-th engaged gear, respectively. The clutch remains locked until the transmitted torque $T_{c}(t)$ exceeds the static friction capacity, $\mu_{s} P_{c}(t)$, where $\mu_{s}$ is the static friction coefficient. The continuous dynamics in location $q_{i}$, with $i=1, \ldots, 5$, and $q_{R}$, are described by a third order linear system

$$
\begin{aligned}
& \dot{x}(t)=A_{i} x(t)+B u(t)+F d(t) \\
& y(t)=C x(t)
\end{aligned}
$$

where

$$
x(t)=\left(\begin{array}{c}
\alpha(t) \\
\omega_{e}(t) \\
\omega_{w}(t)
\end{array}\right), u(t)=T_{e}(t), \quad d(t)=T_{w}(t), \quad y=\left(\begin{array}{c}
\omega_{e}(t) \\
\omega_{w}(t)
\end{array}\right)
$$

and

$$
A_{i}=\left(\begin{array}{ccc}
0 & 1 & -\frac{1}{\tau_{i}} \\
-\frac{k_{i}}{j_{e}} & -\frac{b_{e}+b_{i}}{j_{e}} & \frac{b_{i}}{\tau_{i} j_{e}} \\
\frac{k_{i}}{\tau_{i} j_{w}} & \frac{b_{i}}{\tau_{i} j_{w}} & -\frac{\tau_{i} b_{w}+b_{i}}{\tau_{i} j_{w}}
\end{array}\right), B=\left(\begin{array}{c}
0 \\
\frac{1}{j_{e}} \\
0
\end{array}\right), F=\left(\begin{array}{c}
0 \\
0 \\
-\frac{1}{j_{w}}
\end{array}\right), C=\left(\begin{array}{ccc}
0 & 1 & 0 \\
0 & 0 & 1
\end{array}\right)
$$

where $j_{e}$ is the crankshaft inertia, $j_{w}$ is the vehicle equivalent inertia, $b_{w}$ is the driveline equivalent viscous coefficient and $b_{e}$ is the crankshaft viscous coefficient.

The continuous dynamics in location $q_{N}$, depends on the clutch state (open or slipping) and, when the clutch is slipping, also on the engaged gear. The input events $a_{i}$, with $i=1, \ldots, 5, a_{R}$ and $b$ correspond to internal and exogenous input events. In more detail, the event $b$ is generated when one of the following conditions holds true:

$$
P_{c}(t)=0 \quad \vee \quad\left|T_{c}(t)\right|>\mu_{s} P_{c}(t) \quad \vee \quad \text { lever }=N
$$

and the events $a_{i}$ and $a_{R}$ when all the following conditions are verified

$$
\omega_{e}(t)=\omega_{c}(t) \wedge\left|T_{c}(t)\right| \leq \mu_{s} P_{c}(t) \wedge \text { lever }=i
$$




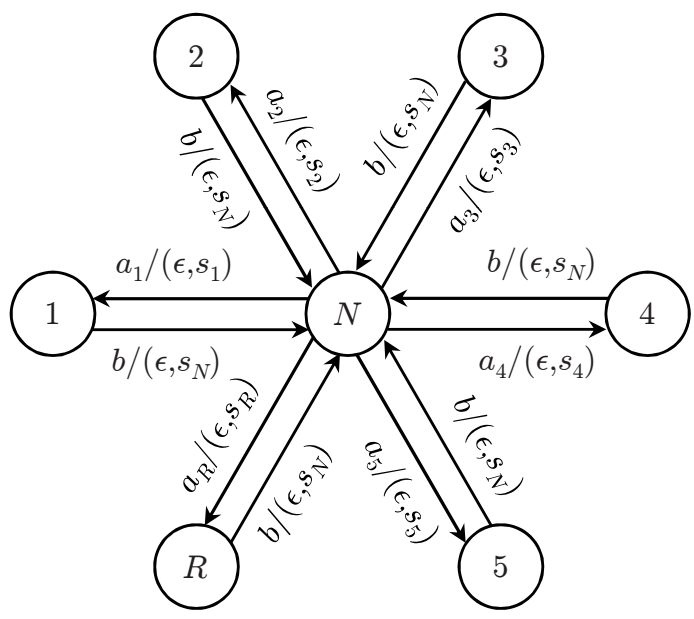

Figure 13: The DES $\hat{\mathcal{D}}_{\mathcal{H}}$ associated to the composition of the driveline hybrid model $\mathcal{H}$ and the transition detector $\mathcal{T}_{\mathcal{H}}$

\subsection{Location observer design}

Since the on-line identification of the actual engaged gear corresponds to the identification of the current location of the driveline hybrid model $\mathcal{H}$, then the hybrid observer design reduces to the design of a location-observer for the hybrid system $\mathcal{H}$.

Consider then the hybrid model $\mathcal{H}$ and note that since the events causing discrete transitions are not measurable, it does not provide any discrete output signal. As a consequence the hybrid model $\mathcal{H}$ is not current-location observable. Assume then that there exists a transition detector $\mathcal{T}_{\mathcal{H}}$ generating the complementary discrete outputs $s_{i}$, with with $i=1, \ldots, 5, s_{R}$ and $s_{N}$ when it detects the transitions to location $q_{i}$, with $i=1, \ldots, 5, q_{R}$ and $q_{N}$, respectively. The complementary DES $\hat{\mathcal{D}}_{\mathcal{H}}$ associated to the composition of the hybrid model $\mathcal{H}$ and the transition detector $\mathcal{T}_{\mathcal{H}}$ is shown in Figure 13. As it can be easily checked, the introduction of the complementary outputs makes the DES $\hat{\mathcal{D}}_{\mathcal{H}}$ current-state observable, that is the hybrid model of the driveline is current-location recoverable with the proposed transition detector. The observer $\hat{\mathcal{D}}_{\mathcal{O}}$ for the DES $\hat{\mathcal{D}}_{\mathcal{H}}$ is depicted in Figure 14.

Transition detector design Recoverability of the hybrid model of the driveline reduces the problem of actual engaged gear identification to the design of the proposed transition detector $\mathcal{T}_{\mathcal{H}}$. This device should be able to generate the complementary outputs $s_{1}, s_{2}, \ldots, s_{5}, s_{R}$ and $s_{N}$ by processing the available inputs and outputs of $\mathcal{H}$, that is the measure of the crankshaft revolution speed $\omega_{e}(t)$, the measure of the wheel revolution speed $\omega_{w}(t)$ and an estimate $\bar{T}_{e}(t)$ of the mean-value of the engine torque $T_{e}(t)$. The proposed scheme is depicted in Figure 15.

Each complementary discrete output $s_{1}, s_{2}, \ldots, s_{5}, s_{R}$ is generated independently from the others by using three cascade blocks: a residual generator, a decision function. and a rising edge detector. 


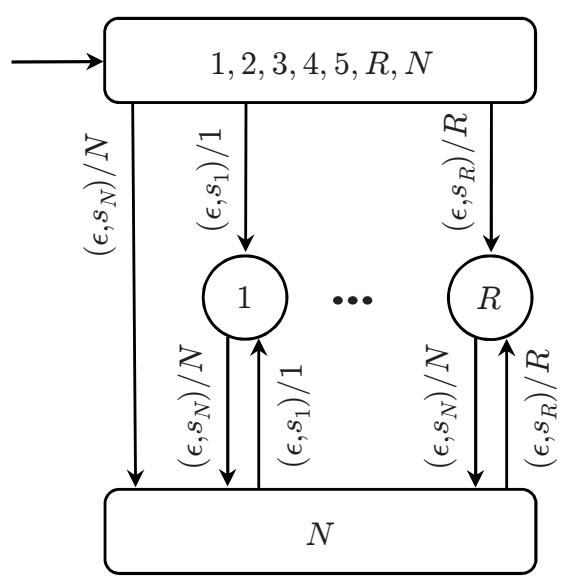

Figure 14: The observer $\hat{\mathcal{D}}_{\mathcal{O}}$.

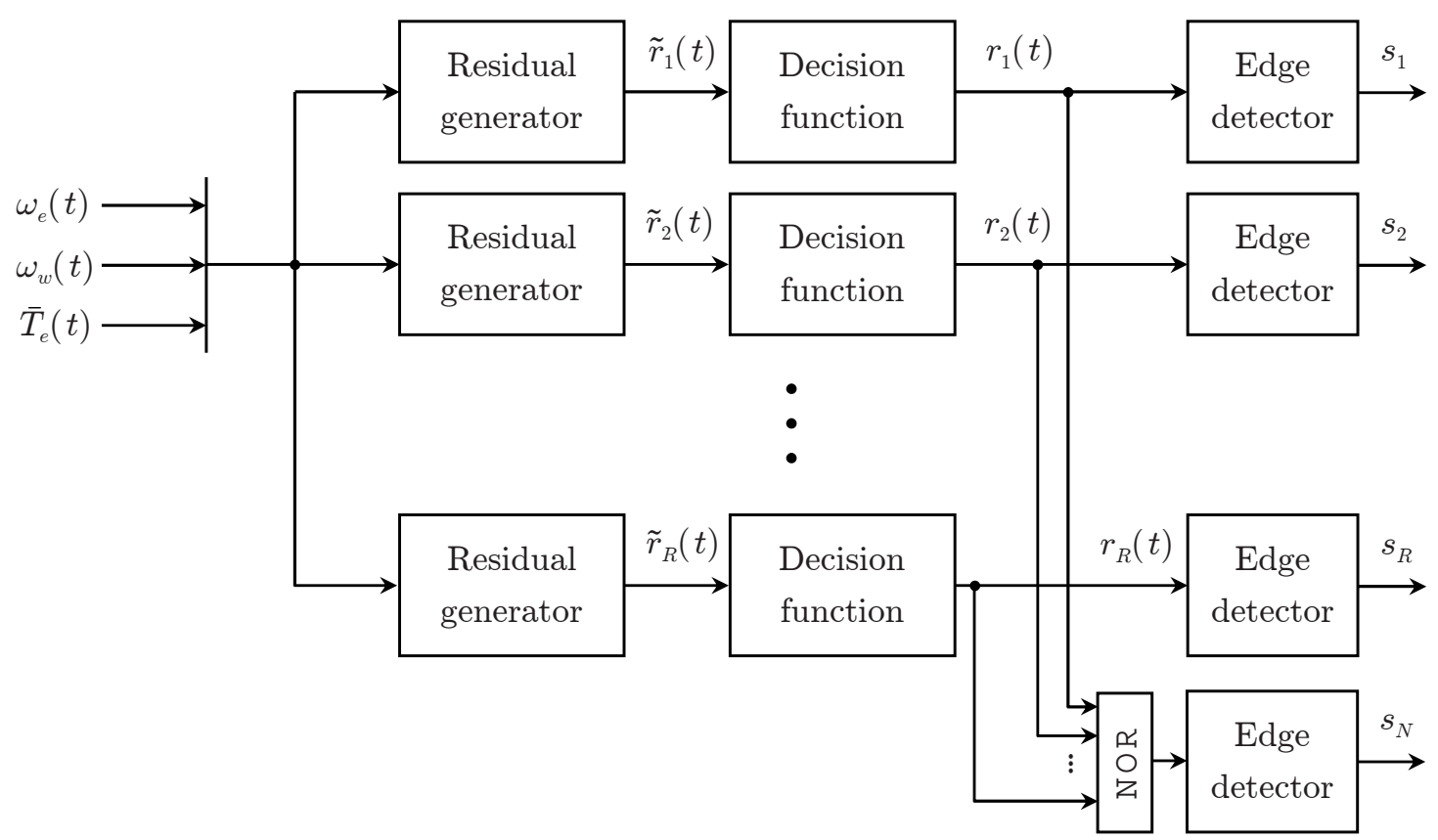

Figure 15: The scheme of the transition detector $\mathcal{T}_{\mathcal{H}}$. 
The $i$-th residual generator produces a residual signal $\tilde{r}_{i}(t)$ converging to zero when the hybrid system is in location $q_{i}$. A simple and reliable approach for this purpose is to use a Luenberger observer, tuned on the continuous time dynamics associated to location $q_{i}$ to be detected:

$$
\begin{aligned}
& \dot{z}_{i}(t)=\left(A_{i}-L_{i} C\right) z_{i}(t)+B u(t)+L_{i} y(t) \\
& \tilde{r}_{i}(t)=C z_{i}(t)-y(t)
\end{aligned}
$$

where $L_{i}$ are design parameters. If the hybrid system is in location $q_{i}$, then the residual $\tilde{r}_{i}(t)$ converges to zero with rate adjustable by $L_{i}$. Such residual $\tilde{r}_{i}(t)$ is affected by several unknown disturbances:

- the quantization error and the delay on the measurements of $\omega_{e}(t)$ and $\omega_{w}(t)$;

- the mismatch between the actual continuous-time and pulsating engine torque $u(t)=T_{e}(t)$ and the event-based estimate $\bar{T}_{e}(t)$, synchronized with engine strokes, used as input of Luenberger observers;

- the not measurable wheel torque $T_{w}(t)$ that acts as a continuous-time disturbance.

The effects of the disturbances on the residual signals have been minimized by appropriately tuning the residuals generators.

The $i$-th decision function outputs a binary signal $r_{i}(t)$, called signatures so that $\tilde{\gamma}=s_{i}$ at the rising edge of $r_{i}(t)$. A very simple implementation is:

$$
r_{i}(t)= \begin{cases}1 & \text { if }\left\|\tilde{r}_{i}(t)\right\| \leq \varepsilon \\ 0 & \text { if }\left\|\tilde{r}_{i}(t)\right\|>\varepsilon\end{cases}
$$

where the threshold $\varepsilon$ is a design parameter. In order to reduce chattering of the signatures $r_{i}(t)$, a more sophisticated decision function has been used: it consists of the cascade of a passive-hysteresis relay and a debouncing algorithm.

Finally, note that location $q_{N}$, representing either driveline open or clutch slipping, cannot be detected using the residual approach. This is because:

- the continuous dynamics associated to this location is more sensitive to torque disturbances, especially during clutch slipping when the clutch plate torque $T_{c}(t)$ is nonzero;

- the disturbances acting on the continuous dynamics cannot be satisfactorily compensated by the residual, being only one output, that is the engine speed, available for feedback.

Hence, the signature $r_{N}(t)$ detecting location $q_{N}$ is obtained by negation of the other, i.e.

$$
r_{N}(t)=\neg\left(r_{1}(t) \vee r_{2}(t) \vee r_{3}(t) \vee r_{4}(t) \vee r_{5}(t) \vee r_{R}(t)\right)
$$

\section{$5.2 \quad$ Experimental results}

The performance of the proposed observer for actual engaged gear identification was tested with experimental data obtained in Magneti Marelli Powertrain using an Opel Astra equipped with a 
Diesel engine and a robotized gearbox SeleSpeed. The experimental data collected the measurements of the estimated engine torque $\bar{T}_{e}(t)$, the crankshaft speed $\omega_{e}(t)$, and the wheel speed $\omega_{w}(t)$ obtained by the engine control unit installed on the vehicle. The specification given by Magneti Marelli Powertrain was to achieve correct identification on a set of maneuvers within a delay of $250 \mathrm{msec}$, using an implementation of the algorithm in discrete-time with a sampling period of 12 msec. The measurements of $\omega_{e}(t)$ and $\omega_{w}(t)$ were affected by delays, but the algorithm proved to be robust with respect to this non ideal situation. For the validation of the identification algorithm, the estimated engaged gear was compared to the signal on actual engaged gear provided by the control unit of the robotized gearbox.

The algorithm was tested on several maneuvers for a total of 250 gear engagements. The actual engaged gear was successfully identified within a delay of $250 \mathrm{msec}$ in $90 \%$ of cases. The unsuccessful cases have been obtained in very critical maneuvers such as gear engagements during sharp braking or clutch abrupt releases. In these cases, the residuals exhibit large oscillations that cause a delay up to $500 \mathrm{msec}$ in the identification. We believe that this delay can be reduced by using a more sophisticated decision function.

Figure 16 reports the results on actual engaged gear identification for the following maneuver: initially the car is at rest, clutch open and first gear engaged. After a clutch slipping phase, the clutch is locked. Later, second gear and then third gear are engaged, passing through idle and clutch slipping. The first plot of the figure shows the residual $\tilde{r}_{N}(t)$ of the idle gear obtained by using a dynamic residual while the second plot shows the residuals $\tilde{r}_{i}(t)$ associated to the gear in engaged state. As the figure makes clear, the residual $\tilde{r}_{N}(t)$ is more affected by noise than the residuals $\tilde{r}_{i}(t)$ and this is due to a larger degree of uncertainty for the friction during idle. This is the reason why the signature $r_{N}(t)$ detecting location $q_{N}$ has been obtained by negation of the other signatures.

Figure 17 shows more in detail the transition from second to third gear. The figure clearly shows that there is a delay in detecting the idle and slipping location. In fact, since the signature $r_{N}(t)$ is obtained by negation of the others, then the location $q_{N}$ is identified only when the residual $\tilde{r}_{2}(t)$ increases over the high value of the relay threshold. The figure also shows that the location $q_{3}$ is identified in advance with respect to the actual transition. To understand this situation, notice that during the slipping phase that precedes each clutch engagement, since the new gear has been already engaged, the continuous dynamics in location $q_{N}$ tends to that of the entering location $q_{i}$ as the slipping decreases. Due to this behavior, transitions from $q_{N}$ to $q_{i}$ never cause abrupt changes in the continuous dynamics. Consequently, the residual norm $\left\|\tilde{r}_{i}(t)\right\|$ gradually decreases during slipping and approaches the low value of the relay threshold before the driveline hybrid model enters location $q_{i}$ (see Figure 17). Hence, occasionally the observer is able to identify the new location $q_{i}$ in advance with respect to the actual transition in the driveline hybrid model.

Finally, the second plot of Figure 17 shows the signal to the input of the debouncing algorithm produced by the passive-hysteresis relay. Notice that, when the residual $\tilde{r}_{3}(t)$ decreases below the low threshold of the relay, the debouncing algorithm waits a time $t_{\text {delay }}$ and then sets the signature $r_{3}(t)$ to 1 only if the residual $\tilde{r}_{3}(t)$ is still below the threshold. 

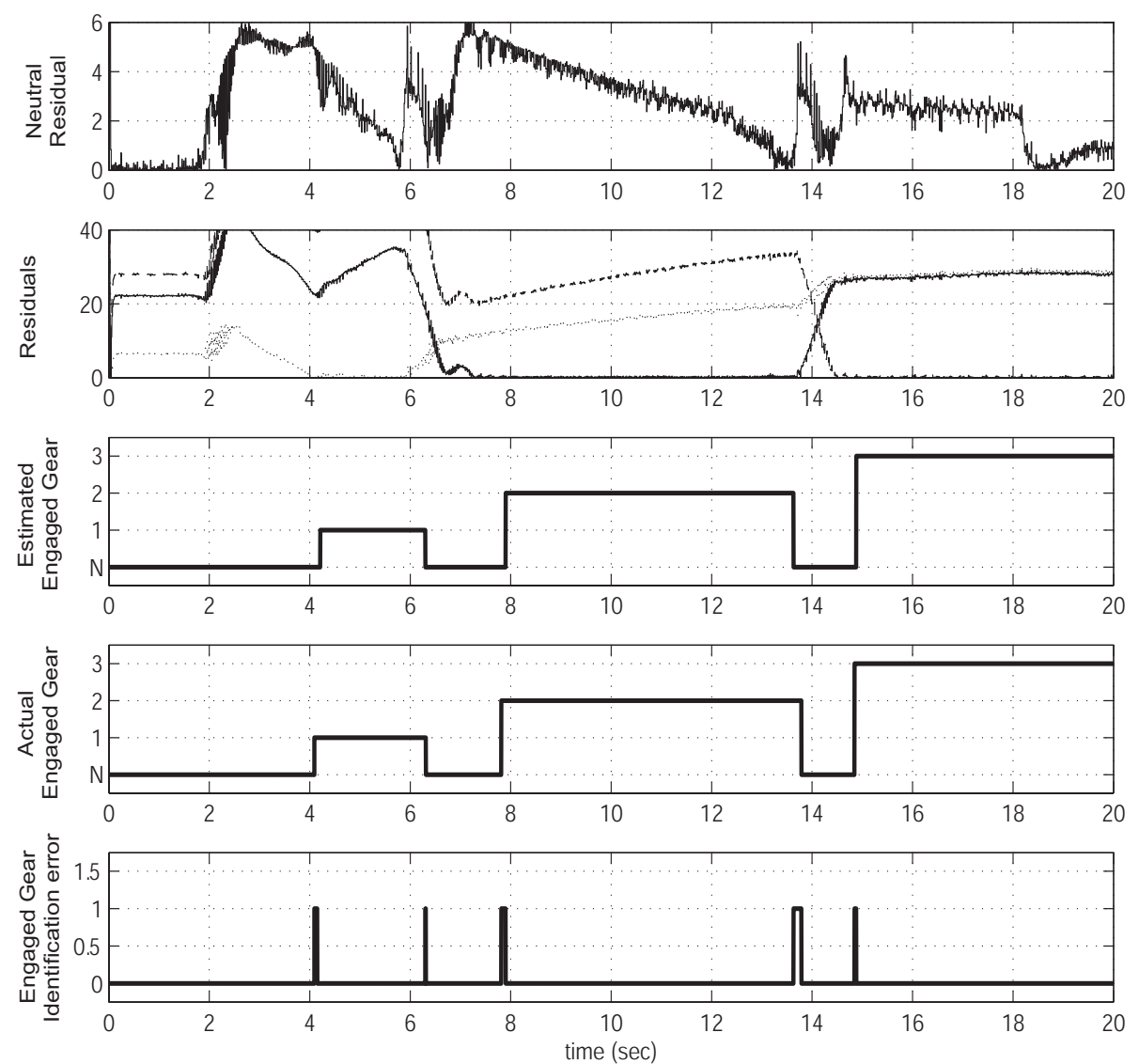

Figure 16: Gear identification with experimental data.

\section{Conclusions}

A methodology for the design of exponentially convergent dynamical observers for hybrid plants with continuous state resets has been presented. The observer consists of two parts: a location observer and a continuous observer providing, respectively, estimates of the plant current location and continuous state. In the design methodology, the interactions between the discrete and the continuous dynamics in the hybrid plant are leveraged to improve convergence performances using:

- additional information from the evolution of the continuous inputs and outputs to identify plant's discrete locations not observable from the discrete inputs and outputs only;

- detection of discrete transitions either to compute the continuous state of the plant at transition times or to reconstruct input-output unobservable components of the continuous plant dynamics. 


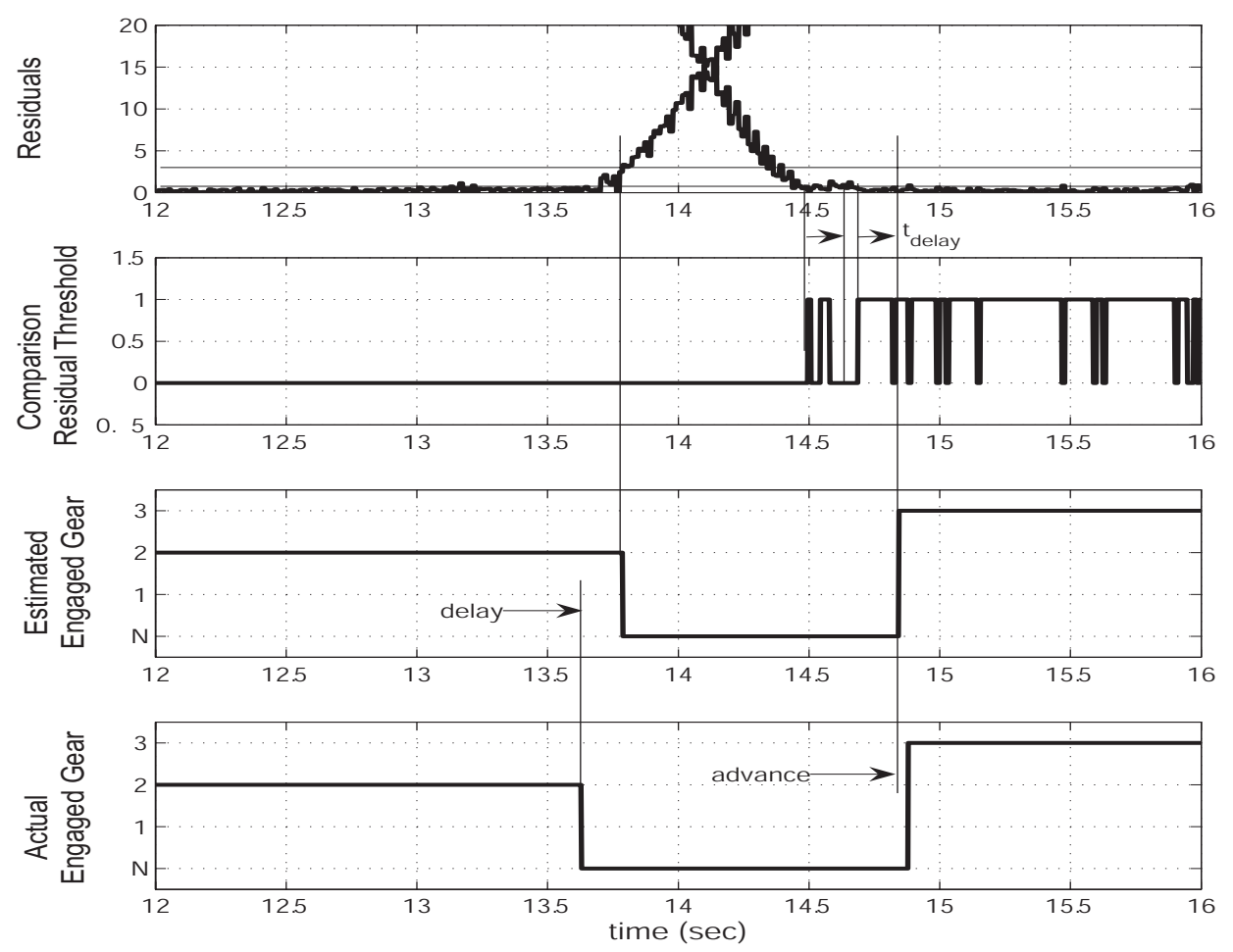

Figure 17: Transition from second to third gear.

A challenging automotive control problem was addressed with the proposed methodology. The synthesized observer was tested with actual, not simulated data obtained for an Opel Astra equipped with a Diesel engine and a robotized gear box. The observer was proven to achieve the performance as specified by Magneti Marelli, a tier one supplier.

As a final remark, we underline that our results on hybrid observer synthesis can be also applied to address the design of diagnosis algorithms. In these applications, the hybrid model describing the behavior of the controlled plant, both in normal operating conditions and partial or full degradation of components, may be very complex due to the high number of locations and transitions, especially in the most critical case of multiple sequential faults. Using our approach results in reducing the diagnosis search space, that is the space of potential models under consideration in each operation mode.

\section{Acknowledgments}

We wish to thank Giovanni Gaviani, Gabriele Serra, Roberto Flora and Eric Massimelli from Magneti Marelli Powertrain (Bologna, Italy) that introduced us to the problems of state estimation in power-train control applications. Moreover, we wish to thank S. Capecce, E. Ceccarelli and M. De Cesare from Magneti Marelli Powertrain (Bologna, Italy) for the collaboration in the experimental 
work and C. Lemma for the work devoted to the design and implementation of the hybrid observer for online identification of the actual engaged gear.

This research was sponsored in part by the MARCO FCRP MUSYC and the Center of Excellence DEWS of Universita' de L'Aquila.

\section{References}

[1] G. A. Ackerson and K. S. Fu. On state estimation in switching environments. IEEE Trans. on Automatic Control, 15(1):10-17, 1970.

[2] A. Alessandri and P. Coletta. Design of luenberger observers for a class of hybrid linear systems. In Hybrid Systems: Computation and Control, volume 2034 of LNCS, pages 7-18. Springer-Verlag, Berlin Heidelberg, 2001.

[3] A. Alessandri and P. Coletta. Switching observers for continuous-time and discrete-time linear systems. In Proceedings of the 2001 American Control Conference, volume 3, pages 2516-21, Arlington, VA, USA, 2001.

[4] A. Balluchi, L. Benvenuti, M. D. Di Benedetto, G. M. Miconi, U. Pozzi, T. Villa, H. Wong-Toi, and A. L. Sangiovanni-Vincentelli. Maximal safe set computation for idle speed control of an automotive engine. In Hybrid Systems: Computation and Control, volume 1790 of Lecture Notes in Computer Science. Springer-Verlag, London, United Kingdom, 2000.

[5] A. Balluchi, L. Benvenuti, M. D. Di Benedetto, C. Pinello, and A. L. Sangiovanni-Vincentelli. Automotive engine control and hybrid systems: Challenges and opportunities. Proceedings of the IEEE, 88, "Special Issue on Hybrid Systems" (invited paper)(7):888-912, July 2000.

[6] A. Balluchi, M. D. Di Benedetto, C. Pinello, C. Rossi, and A. L. Sangiovanni-Vincentelli. Hybrid control in automotive applications: the cut-off control. Automatica, 35, Special Issue on Hybrid Systems:519-535, March 1999.

[7] A. Balluchi, M. D. Di Benedetto, C. Pinello, and A. L. Sangiovanni-Vincentelli. Hybrid control in automotive applications. In D. S. Gilliam G. Picci, editor, Dynamical Systems, Control, Coding, Computer Vision, volume 25 of Progress in Systems and Control Theory, pages 449479. Birkhäuser, Basel, Boston, Berlin, 1999.

[8] A. Bemporad, G., Ferrari-Trecate, and M. Morari. Observability and controllability of piecewise affine and hybrid systems. IEEE Transactions on Automatic Control, 45(10):1864-1876, October 2000.

[9] P. E. Caines, R. Greiner, and S. Wang. Dynamical logic observers for finite automata. In Proceedings of 27th Conference on Decision and Control, pages 226-233, Austin, TX, 1988.

[10] A. Deshpande and P. Varaiya. Viable control of hybrid systems. In Hybrid Systems, volume 999 of Lecture Notes in Computer Science, pages 128-147. Springer, 1994. 
[11] G. Ferrari-Trecate, D. Mignone, and M. Morari. Moving horizon estimation for hybrid systems. In Proceedings of the 2000 American Control Conference, volume 3, pages 1684-8, Chicago, IL, USA, 2000.

[12] M. Fliess, C. Join, and W. Perruquetti. Real-time estimation for switched linear systems. In 47th IEEE Conference on Decision and Control, Cancun, Mexico, 2008.

[13] J.P. Hespanha and A.S. Morse. Stability of switched systems with average dwell-time. In Proceedings of the 38th IEEE Conference on Decision and Control, CDC1999, volume 3, pages 2655-60, Phoenix, AZ, USA, December 1999.

[14] M.W. Hofbaur and B.C. Williams. Mode estimation of probabilistic hybrid systems. In C.J. Tomlin and M.R. Greenstreet, editors, Hybrid Systems: Computation and Control, volume 2289, pages 253-266. Springer-Verlag, 2002.

[15] R.E. Kalman. A new approach to liner filtering and prediction problems. Transactions of the ASME - Journal of Basic Engineering, D:35-45, 1960.

[16] C. Van Loan. The sensitivity of the matrix exponential. SIAM Journal of Number Analysis, 14(6):971-981, December 1977.

[17] D.G. Luenberger. An introduction to observers. IEEE Transactions on Automatic Control, 16(6):596-602, Dec 1971.

[18] M.-A. Massoumnia, G.C., Verghese, and A.S. Willsky. Failure detection and identification. IEEE Transactions on Automatic Control, 34(3):316-21, March 1989.

[19] S. McIlraith, G. Biswas, D. Clancy, and V. Gupta. Hybrid systems diagnosis. In Nancy Lynch and Bruce H. Krogh (Eds.), editors, Hybrid Systems: Computation and Control, volume 1790, pages 282-295. Springer-Verlag, 2000.

[20] A.S. Morse. Supervisory control of a families of linear set-point controllers - part 1: Exact matching. IEEE Trans. on Automatic Control, 41(10):1413-1431, October 1996.

[21] P.J. Mosterman and G. Biswas. Building hybrid observers for complex dynamic systems using model abstractions. In F. Vaandrager and J. van Schuppen, editors, Hybrid Systems: Computation and Control, volume 1569, pages 178-192. Springer-Verlag, 1999.

[22] S. Narasimhan, G. Biswas, G. Karsai, T. Pasternak, and F. Zhao. Building observers to address fault isolation and control problems in hybrid dynamic systems. In Proceedings of IEEE International Conference on Systems, Man, and Cybernetics, volume 4, pages 2393-8, Nashville, TN, USA, 2000.

[23] C. M. Özveren and A. S. Willsky. Observability of discrete event dynamic systems. IEEE Trans. on Automatic Control, 35(7):797-806, July 1990.

[24] S. Pettersson. Designing switched observers for switched systems using multiple Lyapunov functions and dwell-time switching. In 2nd IFAC Conf. on Analysis and Design of Hybrid Systems, pages 18-23, Alghero, Italy, 2006. 
[25] P. J. Ramadge. Observability of discrete event-systems. In Proceedings of 25th Conference on Decision and Control, pages 1108-1112, Athens, Greece, 1986.

[26] E. De Santis, M.D.Di Benedetto, and G. Pola: A structural approach to detectability for a class of hybrid systems. Automatica, 45:2002-2009, 2009.

[27] A. Tanwani, H. Shim, and D. Liberzon. Observability implies observer design for switched linear systems. In Proceedings of the 14th international conference on Hybrid systems: computation and control, HSCC '11, pages 3-12, New York, NY, USA, 2011. ACM.

[28] M. Vidyasagar. Nonlinear Systems Analysis. Prentice-Hall, Inc, Englewood Cliffs, N.J., 1978.

[29] J. Zhang, K.H. Johansson, J. Lygeros, and S. Sastry. Zeno hybrid systems. International Journal of Robust and Nonlinear Control, 11:435-451, 2001.

[30] Q. Zhang. Hybrid filtering for linear systems with non-gaussian disturbances. IEEE Trans. on Automatic Control, 45(1):50-61, 2000.

\section{Appendix}

The following results have been used in the proof of Theorems 6 and 7 :

Lemma 8 ([16]) Let $A \in \mathbb{R}^{n \times n}$ be a matrix with distinct eigenvalues. Then

$$
\left\|e^{A t}\right\| \leq k(A) e^{\alpha(A) t} \quad \forall t \geq 0
$$

If $F \in \mathbb{R}^{n \times n}$ is another matrix with distinct eigenvalues and $\alpha(F)<\alpha(A)<0$, then

$$
\begin{aligned}
\left\|I-e^{A t}\right\| & \leq k(A)\|A\| t \quad \forall t \geq 0 \\
\left\|e^{F t}-e^{A t}\right\| & \leq k(F) k(A)(\|F\|+\|A\|) t \quad \forall t \geq 0
\end{aligned}
$$

\section{Proof of Theorem 6.}

Let us introduce the following notation (see [28]): $\|M\|_{\infty}$ and $\|M\|_{1}$ the $L^{\infty}$ and $L^{1}$-norm of a matrix $M$, respectively.

From the hypothesis of current-location observability of the hybrid system $\mathcal{H}$ it follows that there exists a positive integer $K$ such that the current location of the hybrid plant is properly identified for any $k \geq K$. In order to prove exponentially ultimately boundedness of the proposed hybrid observer $\mathcal{H}_{O}$ according to definition 2, we consider the evolution of the observation error $\zeta(t)$ for $t \geq t_{K}$.

Consider then two subsequent transitions of the hybrid system $\mathcal{H}$, occurring at times $t_{k-1}$ and $t_{k}$ respectively, with $t_{k}>t_{k-1} \geq t_{K}$. Let $\tilde{q}=\left\{q_{i}\right\} \in P$ for $t \in\left[t_{k-1}, t_{k}\right)$ and $\tilde{q}=\left\{q_{j}\right\} \in P$ for $t \in\left[t_{k}, t_{k+1}\right)$. Let also $F_{i}=A_{i}-G_{i} C_{i}$. From (18) and (19), we have

$$
\begin{aligned}
\zeta(t) & =e^{F_{i}\left(t-t_{k-1}\right)} \zeta\left(t_{k-1}\right)-\int_{0}^{t-t_{k-1}} e^{F_{i}\left(t-t_{k-1}-\tau\right)} w\left(\tau+t_{k-1}\right) d \tau \quad \text { for } t \in\left[t_{k-1}, t_{k}\right) \\
\zeta\left(t_{k}\right) & =R_{i j}^{1} \zeta\left(t_{k}^{-}\right)
\end{aligned}
$$


Since by (14)

$$
\sup _{t \geq t_{k-1}}\left\|\int_{0}^{t-t_{k-1}} e^{F_{i}\left(t-t_{k-1}-\tau\right)} w\left(\tau+t_{k-1}\right) d \tau\right\| \leq \sqrt{n}\left\|e^{F_{i} t}\right\|_{1}\|w(t)\|_{\infty} \leq \sqrt{n}\left\|e^{F_{i} t}\right\|_{1} W,
$$

then by (24) we have

$$
\begin{aligned}
\|\zeta(t)\| & \leq k\left(F_{i}\right) e^{\alpha\left(F_{i}\right)\left(t-t_{k-1}\right)}\left\|\zeta\left(t_{k-1}\right)\right\|+\sqrt{n}\left\|e^{F_{i} t}\right\|_{1} W \quad \forall t \in\left[t_{k-1}, t_{k}\right) \\
\left\|\zeta\left(t_{k}^{-}\right)\right\| & \leq k\left(F_{i}\right) e^{\alpha\left(F_{i}\right)\left(t_{k}-t_{k-1}\right)}\left\|\zeta\left(t_{k-1}\right)\right\|+\sqrt{n}\left\|e^{F_{i} t}\right\|_{1} W \\
\left\|\zeta\left(t_{k}\right)\right\| & \leq r_{i}^{1} k\left(F_{i}\right) e^{\alpha\left(F_{i}\right)\left(t_{k}-t_{k-1}\right)}\left\|\zeta\left(t_{k-1}\right)\right\|+c_{i} W
\end{aligned}
$$

where $c_{i}=\sqrt{n} r_{i}^{1}\left\|e^{F_{i} t}\right\|_{1}$ and (29) yields for any $\left\{q_{j}\right\}$ such that $q_{j} \in \operatorname{Reach}\left(q_{i}\right)$.

Moreover, since by the dwell time hypothesis $t_{k}-t_{k-1} \geq D$, then for any rate of convergence $\mu$ and sufficiently large $D$, there always exist parameters $G_{i}$ such that

$$
\alpha\left(A_{i}-G_{i} C_{i}\right)+\frac{\max \left\{0, \log \left[r_{i}^{1} k\left(A_{i}-G_{i} C_{i}\right)\right]\right\}}{D} \leq-\mu<0,
$$

As a consequence,

$r_{i}^{1} k\left(F_{i}\right) e^{\alpha\left(F_{i}\right)\left(t_{k}-t_{k-1}\right)}=e^{\log \left[r_{i}^{1} k\left(F_{i}\right)\right]+\alpha\left(F_{i}\right)\left(t_{k}-t_{k-1}\right)} \leq e^{\left[\alpha\left(F_{i}\right)+\frac{\max \left\{0, \log \left[r_{i}^{1} k\left(F_{i}\right)\right]\right\}}{D}\right]\left(t_{k}-t_{k-1}\right)} \leq e^{-\mu\left(t_{k}-t_{k-1}\right)}$

Hence, from (29),

$$
\left\|\zeta\left(t_{k}\right)\right\| \leq e^{-\mu\left(t_{k}-t_{k-1}\right)}\left\|\zeta\left(t_{k-1}\right)\right\|+c W \leq e^{-\mu\left(t_{k}-t_{K}\right)}\left\|\zeta\left(t_{K}\right)\right\|+c W \sum_{h=K}^{k} e^{-\mu\left(t_{k}-t_{h}\right)}
$$

where $c=\max _{\left\{q_{i}\right\} \in P}\left\{c_{i}\right\}$. Finally, since $t_{k}-t_{h} \geq(k-h) D$ and $e^{-\mu D}<1$, then

$$
\left\|\zeta\left(t_{k}\right)\right\| \leq e^{-\mu\left(t_{k}-t_{K}\right)}\left\|\zeta\left(t_{K}\right)\right\|+c W \sum_{h=K}^{k} e^{-\mu(k-h) D}=e^{-\mu\left(t_{k}-t_{K}\right)}\left\|\zeta\left(t_{K}\right)\right\|+\frac{c W}{1-e^{-\mu D}}
$$

This shows that the value of the norm of the observation error after each transition is upper bounded by an exponential with rate $-\mu$ that converges to the value $\frac{c W}{1-e^{-\mu D}}$. Moreover, substituting the upper bound (30) for $\left\|\zeta\left(t_{k}\right)\right\|$ into (28), we get

$$
\begin{aligned}
\|\zeta(t)\| \leq & k\left(F_{i}\right) e^{\alpha\left(F_{i}\right)\left(t-t_{k-1}\right)} e^{-\mu\left(t_{k-1}-t_{K}\right)}\left\|\zeta\left(t_{K}\right)\right\|+k\left(F_{i}\right) e^{\alpha\left(F_{i}\right)\left(t-t_{k-1}\right)} \frac{c W}{1-e^{-\mu D}} \\
& +\sqrt{n}\left\|e^{F_{i} t}\right\|_{1} W \leq k\left(F_{i}\right) e^{-\mu\left(t-t_{K}\right)}\left\|\zeta\left(t_{K}\right)\right\|+\left[\frac{k\left(F_{i}\right) c}{1-e^{-\mu D}}+\sqrt{n}\left\|e^{F_{i} t}\right\|_{1}\right] W
\end{aligned}
$$

for every $t \in\left[t_{k-1}, t_{k}\right)$ where $q=q_{i}$. Finally,

$$
\|\zeta(t)\| \leq \max _{\left\{q_{i}\right\} \in P}\left\{k\left(F_{i}\right)\right\} e^{-\mu\left(t-t_{K}\right)}\left\|\zeta\left(t_{K}\right)\right\|+\max _{\left\{q_{i}\right\} \in P}\left\{\frac{k\left(F_{i}\right) c}{1-e^{-\mu D}}+\sqrt{n}\left\|e^{F_{i} t}\right\|_{1}\right\} W
$$


for every $t \geq t_{K}$ and for any switching sequence of plant locations. This proves exponentially ultimately boundedness of the hybrid observer under the action of the bounded disturbance $w(t)$. Moreover, in the case of absence of disturbance, the hybrid observer is exponentially convergent as shown by (31) with $W=0$.

Proof of Theorem 7. Let $B_{x}, B_{u}>0$ such that $\|x(t)\|_{\infty} \leq B_{x}$ and $\|u(t)\|_{\infty} \leq B_{u}$, so that

$$
\left\|v_{j i}(t)\right\|_{\infty} \leq V_{j i} \quad \forall\left\{q_{j}\right\} \in P \text { and } q_{i} \in \operatorname{Reach}\left(q_{j}\right)
$$

with $V_{j i}=\left\|\left[\left(A_{j}-A_{i}\right)-G_{j}\left(C_{j}-C_{i}\right)\right]\right\|_{\infty} B_{x}+\left\|B_{j}-B_{i}\right\|_{\infty} B_{u}$.

Consider two subsequent transitions of the hybrid system $\mathcal{H}$, occurring at times $t_{k-1}$ and $t_{k}$ respectively.

Let $q=q_{j}$ for $t \in\left[t_{k-1}, t_{k}\right)$ and $q=q_{i} \in \operatorname{Reach}\left(q_{j}\right)$ for $t \in\left[t_{k}, t_{k+1}\right)$. By the hypothesis on dwell time equal to $D, t_{k}-t_{k-1} \geq D$. Moreover, by hypothesis, the transition detector is able to detect any transition of $\mathcal{H}$ for wich a complementary output has to be produced within a time $\Delta<D$. Let $\beta=D-\Delta>0$. Then the location observer identifies the $k-1$-th and $k+1$-th state transitions at some times $\hat{t}_{k-1}$ and $\hat{t}_{k}$, respectively, with $0 \leq \hat{t}_{k-1}-t_{k-1} \leq \Delta$ and $0 \leq \hat{t}_{k}-t_{k} \leq \Delta$. That is: $\tilde{q}=\left\{q_{j}\right\}$ for $t \in\left[\hat{t}_{k-1}, \hat{t}_{k}\right)$ and $\tilde{q}=\left\{q_{i}\right\}$ for $t \in\left[\hat{t}_{k}, \hat{t}_{k+1}\right)$. Furthermore, notice that, by the hypothesis on the dwell time and on the transition detector, $\hat{t}_{k}-\hat{t}_{k-1} \geq t_{k}-\hat{t}_{k-1} \geq D-\Delta=\beta>0$.

Consider the time interval $\left[\hat{t}_{k-1}, \hat{t}_{k}\right)$. The composed hybrid system $\mathcal{H} \otimes \mathcal{H}_{O}$ lies on location $\left(q_{j},\left\{q_{j}\right\}\right)$ for $t \in\left[\hat{t}_{k-1}, t_{k}\right)$, and location $\left(q_{i},\left\{q_{j}\right\}\right)$ for $t \in\left[t_{k}, \hat{t}_{k}\right)$. The observation error continuos dynamics are the following:

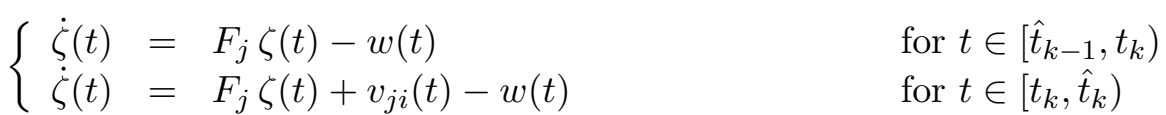

where $F_{j}=A_{j}-G_{j} C_{j}$, while the resets of the observation error are the following:

$$
\begin{gathered}
\zeta\left(t_{k}\right)=\zeta\left(t_{k}^{+}\right)=\zeta\left(t_{k}^{-}\right)-R_{j i}^{0}+\left[I-R_{j i}^{1}\right] x\left(t_{k}^{-}\right) \quad t=t_{k} \\
\zeta\left(\hat{t}_{k}\right)=\zeta\left(\hat{t}_{k}^{+}\right)=R_{j i}^{1} \zeta\left(\hat{t}_{k}^{-}\right)+R_{j i}^{0}-\left[I-R_{j i}^{1}\right] x\left(\hat{t}_{k}\right) \quad t=\hat{t}_{k}
\end{gathered}
$$

Since in the time interval $\left[\hat{t}_{k-1}, t_{k}\right)$ the location observer properly identifies the plant location $q_{j}$, then the observation error $\zeta(t)$ is subject to an autonomous dynamic that convergences to zero if the matrix $F_{j}$ is Hurwitz for some choice of the observer gain $G_{j}$.

In particular, for any rate of convergence $\mu$ and sufficiently large $D$, there always exist parameters $G_{j}$ such that

$$
\alpha\left(A_{j}-G_{j} C_{j}\right)+\frac{\max \left\{0, \log \left[r_{j}^{1} k\left(A_{j}-G_{j} C_{j}\right)\right]\right\}}{\beta} \leq-\mu<0,
$$

However, due to the mismatch between the plant location and the observer location for $t \in$ $\left[t_{k}, \hat{t}_{k}\right), \zeta(t)$ may fail to converge later due to the injection of the disturbance $v_{j i}(t)$ in the evolution of $\zeta(t)$. 
Hence, the convergent behavior for $t \in\left[\hat{t}_{k-1}, t_{k}\right)$ has to compensate the divergent behavior for $t \in\left[t_{k}, \hat{t}_{k}\right)$. By integrating (32), we have

$$
\zeta(t)=e^{F_{j}\left(t-\hat{t}_{k-1}\right)} \zeta\left(\hat{t}_{k-1}\right)-\int_{0}^{t-\hat{t}_{k-1}} e^{F_{j}\left(t-\hat{t}_{k-1}-\tau\right)} w\left(\tau+\hat{t}_{k-1}\right) d \tau \quad \forall t \in\left[\hat{t}_{k-1}, t_{k}\right)
$$

and, by (33),

$$
\begin{aligned}
\zeta\left(t_{k}\right) & =\zeta\left(t_{k}^{-}\right)-R_{j i}^{0}+\left[I-R_{j i}^{1}\right] x\left(t_{k}^{-}\right) \\
& =e^{F_{j}\left(t_{k}-\hat{t}_{k-1}\right)} \zeta\left(\hat{t}_{k-1}\right)-\int_{0}^{t_{k}-\hat{t}_{k-1}} e^{F_{j}\left(t_{k}-\hat{t}_{k-1}-\tau\right)} w\left(\tau+\hat{t}_{k-1}\right) d \tau-R_{j i}^{0}+\left[I-R_{j i}^{1}\right] x\left(t_{k}^{-}\right)
\end{aligned}
$$

Hence, by integrating (32), for $t \in\left[t_{k}, \hat{t}_{k}\right)$ we have

$$
\begin{aligned}
\zeta(t)= & e^{F_{j}\left(t-t_{k}\right)} \zeta\left(t_{k}\right)-\int_{0}^{t-t_{k}} e^{F_{j}\left(t-t_{k}-\tau\right)} w\left(\tau+t_{k}\right) d \tau+\int_{0}^{t-t_{k}} e^{F_{j}\left(t-t_{k}-\tau\right)} v_{j i}\left(\tau+t_{k}\right) d \tau \\
= & e^{F_{j}\left(t-\hat{t}_{k-1}\right)} \zeta\left(\hat{t}_{k-1}\right)-\int_{0}^{t-\hat{t}_{k-1}} e^{F_{j}\left(t-\hat{t}_{k-1}-\tau\right)} w\left(\tau+\hat{t}_{k-1}\right) d \tau-e^{F_{j}\left(t-t_{k}\right)} R_{j i}^{0} \\
& +e^{F_{j}\left(t-t_{k}\right)}\left[I-R_{j i}^{1}\right] x\left(t_{k}^{-}\right)+\int_{0}^{t-t_{k}} e^{F_{j}\left(t-t_{k}-\tau\right)} v_{j i}\left(\tau+t_{k}\right) d \tau \quad \forall t \in\left[t_{k}, \hat{t}_{k}\right)
\end{aligned}
$$

Finally, by (34)

$$
\begin{aligned}
\zeta\left(\hat{t}_{k}\right)= & R_{j i}^{1} \zeta\left(\hat{t}_{k}^{-}\right)+R_{j i}^{0}-\left[I-R_{j i}^{1}\right] e^{A_{i}\left(\hat{t}_{k}-t_{k}\right)} x\left(t_{k}\right) \\
& \quad-\left[I-R_{j i}^{1}\right] \int_{0}^{\hat{t}_{k}-t_{k}} e^{A_{i}\left(\hat{t}_{k}-t_{k}-\tau\right)} B_{i} u\left(\tau+t_{k}\right) d \tau-\left[I-R_{j i}^{1}\right] \int_{0}^{\hat{t}_{k}-t_{k}} e^{A_{i}\left(\hat{t}_{k}-t_{k}-\tau\right)} w\left(\tau+t_{k}\right) d \tau \\
= & R_{j i}^{1} e^{F_{j}\left(\hat{t}_{k}-\hat{t}_{k-1}\right)} \zeta\left(\hat{t}_{k-1}\right)-R_{j i}^{1} \int_{0}^{\hat{t}_{k}-\hat{t}_{k-1} e^{F_{j}\left(\hat{t}_{k}-\hat{t}_{k-1}-\tau\right)} w\left(\tau+\hat{t}_{k-1}\right) d \tau} \\
& +\left[\left(I-e^{A_{i}\left(\hat{t}_{k}-t_{k}\right)}\right)-R_{j i}^{1}\left(e^{F_{j}\left(\hat{t}_{k}-t_{k}\right)}-e^{A_{i}\left(\hat{t}_{k}-t_{k}\right)}\right)\right] R_{j i}^{0} \\
& +\left\{\left[I-R_{j i}^{1}\right]\left(e^{F_{j}\left(\hat{t}_{k}-t_{k}\right)}-e^{A_{i}\left(\hat{t}_{k}-t_{k}\right)}\right) R_{j i}^{1}+\left[\left(I-e^{F_{j}\left(\hat{t}_{k}-t_{k}\right)}\right) R_{j i}^{1}-R_{j i}^{1}\left(I-e^{F_{j}\left(\hat{t}_{k}-t_{k}\right)}\right)\right]\right\} x\left(t_{k}^{-}\right) \\
& +R_{j i}^{1} \int_{0}^{\hat{t}_{k}-t_{k}} e^{F_{j}\left(\hat{t}_{k}-t_{k}-\tau\right)} v_{j i}\left(\tau+t_{k}\right) d \tau-R_{j i}^{1} \int_{0}^{\hat{t}_{k}-\hat{t}_{k-1}} e^{F_{j}\left(\hat{t}_{k}-\hat{t}_{k-1}-\tau\right)} w\left(\tau+\hat{t}_{k-1}\right) d \tau \\
& -\left[I-R_{j i}^{1}\right] \int_{0}^{\hat{t}_{k}-t_{k}} e^{A_{i}\left(\hat{t}_{k}-t_{k}-\tau\right)} B_{i} u\left(\tau+t_{k}\right) d \tau-\left[I-R_{j i}^{1}\right] \int_{0}^{\hat{t}_{k}-t_{k}} e^{A_{i}\left(\hat{t}_{k}-t_{k}-\tau\right)} w\left(\tau+t_{k}\right) d \tau \quad(37)
\end{aligned}
$$

Then, an upper bound for the norm of the observation error $\|\zeta\|$ at each time $\hat{t}_{k}$ is obtained as follows. Since the observation matrices $F_{j}=A_{j}-G_{j} C_{j}$ can be chosen in order to have distinct eigenvalues, then by (24) we have

$$
\left\|R_{j i}^{1} e^{F_{j}\left(\hat{t}_{k}-\hat{t}_{k-1}\right)} \zeta\left(\hat{t}_{k-1}\right)\right\| \leq r_{j}^{1} k\left(F_{j}\right) e^{\alpha\left(F_{j}\right)\left(\hat{t}_{k}-\hat{t}_{k-1}\right)}\left\|\zeta\left(\hat{t}_{k-1}\right)\right\|
$$


Moreover, since $\hat{t}_{k}-\hat{t}_{k-1} \geq \beta$, then it follows that

$r_{j}^{1} k\left(F_{j}\right) e^{\alpha\left(F_{j}\right)\left(\hat{t}_{k}-\hat{t}_{k-1}\right)}=e^{\log \left[r_{j}^{1} k\left(F_{j}\right)\right]+\alpha\left(F_{j}\right)\left(\hat{t}_{k}-\hat{t}_{k-1}\right)} \leq e^{\left[\alpha\left(F_{j}\right)+\frac{\max \left\{0, \log \left[r_{j}^{1} k\left(F_{j}\right)\right]\right\}}{\beta}\right]\left(\hat{t}_{k}-\hat{t}_{k-1}\right)} \leq e^{-\mu\left(\hat{t}_{k}-\hat{t}_{k-1}\right)}$

Hence,

$$
\left\|R_{j i}^{1} e^{F_{j}\left(\hat{t}_{k}-\hat{t}_{k-1}\right)} \zeta\left(\hat{t}_{k-1}\right)\right\| \leq e^{-\mu\left(\hat{t}_{k}-\hat{t}_{k-1}\right)}\left\|\zeta\left(\hat{t}_{k-1}\right)\right\|
$$

By (25-26), terms right multiplied by $R_{j i}^{0}$ and $x\left(t_{k}^{-}\right)$are upper bounded by

$$
\begin{aligned}
& {\left[k\left(A_{i}\right)\left\|A_{i}\right\|+k\left(A_{i}\right) k\left(F_{j}\right)\left(\left\|A_{i}\right\|+\left\|F_{j}\right\|\right)\left\|R_{j i}^{1}\right\|\right]\left\|R_{j i}^{0}\right\| \Delta=b_{j i}^{1} \Delta} \\
& {\left[k\left(A_{i}\right) k\left(F_{j}\right)\left(\left\|A_{i}\right\|+\left\|F_{j}\right\|\right)\left\|I-R_{j i}^{1}\right\|\left\|R_{j i}^{1}\right\|+2 k\left(F_{j}\right)\left\|F_{j}\right\|\left\|R_{j i}^{1}\right\|\right] B_{x} \Delta=b_{j i}^{2} B_{x} \Delta}
\end{aligned}
$$

respectively. Furthermore, for the $v_{j i}$ forced term, since $\hat{t}_{k}-t_{k} \leq \Delta$, then

$$
\begin{aligned}
& \left\|R_{j i}^{1} \int_{0}^{\hat{t}_{k}-t_{k}} e^{F_{j}\left(\hat{t}_{k}-t_{k}-\tau\right)} v_{j i}\left(\tau+t_{k}\right) d \tau\right\| \leq\left\|R_{j i}^{1}\right\| k\left(F_{j}\right) \int_{0}^{\hat{t}_{k}-t_{k}} e^{\alpha\left(F_{j}\right)\left(\hat{t}_{k}-t_{k}-\tau\right)}\left\|v\left(\tau+t_{k}\right)\right\| d \tau \\
& \leq\left\|R_{j i}^{1}\right\| k\left(F_{j}\right) \sup _{t \geq 0}\left\|v_{j i}(t)\right\| \int_{0}^{\hat{t}_{k}-t_{k}} e^{\alpha\left(F_{j}\right) \tau} d \tau \leq\left\|R_{j i}^{1}\right\| \sqrt{n} k\left(F_{j}\right)\left\|v_{j i}(t)\right\|_{\infty} \frac{e^{\alpha\left(F_{j}\right)\left(\hat{t}_{k}-t_{k}\right)}-1}{\alpha\left(F_{j}\right)} \\
& \leq\left\|R_{j i}^{1}\right\| \sqrt{n} k\left(F_{j}\right) V_{j i} \Delta=b_{j i}^{3} V_{j i} \Delta
\end{aligned}
$$

Moreover, as in (27),

$$
\left\|R_{j i}^{1} \int_{0}^{\hat{t}_{k}-\hat{t}_{k-1}} e^{F_{j}\left(\hat{t}_{k}-\hat{t}_{k-1}-\tau\right)} w\left(\tau+\hat{t}_{k-1}\right) d \tau\right\| \leq\left\|R_{j i}^{1}\right\| \sqrt{n}\left\|e^{F_{j} t}\right\|_{1} W=c_{i j} W
$$

Finally, by the same arguments of (41), the $u(t)$ and $w(t)$ forced term are bounded by

$$
\begin{aligned}
\left\|I-R_{j i}^{1}\right\| \sqrt{n} k\left(A_{i}\right)\left\|B_{i}\right\| B_{u} \Delta & =b_{j i}^{4} B_{u} \Delta \\
\left\|I-R_{j i}^{1}\right\| \sqrt{n} k\left(A_{i}\right) W \Delta & =d_{j i} W \Delta
\end{aligned}
$$

By using (38-44), the norm of the observation error is upper bounded at each observer transition time $\hat{t}_{k}$ as follows:

$$
\left\|\zeta\left(\hat{t}_{k}\right)\right\| \leq e^{-\mu\left(\hat{t}_{k}-\hat{t}_{k-1}\right)}\left\|\zeta\left(\hat{t}_{k-1}\right)\right\|+\left[b_{j i}^{1}+b_{j i}^{2} B_{x}+b_{j i}^{3} V_{j i}+b_{j i}^{4} B_{u}\right] \Delta+c_{j i} W+d_{j i} W \Delta
$$

Introduce

$$
b=\max _{\left\{q_{j}\right\} \in P} \max _{q_{i} \in \operatorname{Reach}\left(q_{j}\right)}\left\{b_{j i}^{1}+b_{j i}^{2} B_{x}+b_{j i}^{3} V_{j i}+b_{j i}^{4} B_{u}\right\},
$$

$c=\max _{\left\{q_{j}\right\} \in P} \max _{q_{i} \in \operatorname{Reach}\left(q_{j}\right)}\left\{c_{j i}\right\}$, and $d=\max _{\left\{q_{j}\right\} \in P} \max _{q_{i} \in \operatorname{Reach}\left(q_{j}\right)}\left\{d_{j i}\right\}$.

Since $\hat{t}_{k}-\hat{t}_{k-1} \geq \beta$ and $e^{-\mu \beta}<1$, then from (45) we obtain

$\left\|\zeta\left(\hat{t}_{k}\right)\right\| \leq e^{-\mu\left(\hat{t}_{k}-\hat{t}_{k-1}\right)}\left\|\zeta\left(\hat{t}_{k-1}\right)\right\|+b \Delta+c W+d W \Delta \leq e^{-\mu\left(\hat{t}_{k}-\hat{t}_{K}\right)}\left\|\zeta\left(\hat{t}_{K}\right)\right\|+(b \Delta+c W+d W \Delta) \sum_{h=K}^{k} e^{-\mu\left(\hat{t}_{k}-\hat{t}_{h}\right)}$ 
$\leq e^{-\mu\left(\hat{t}_{k}-\hat{t}_{K}\right)}\left\|\zeta\left(\hat{t}_{K}\right)\right\|+(b \Delta+c W+d W \Delta) \sum_{h=-\infty}^{k} e^{-\mu(k-h) \beta}=e^{-\mu\left(\hat{t}_{k}-\hat{t}_{K}\right)}\left\|\zeta\left(\hat{t}_{K}\right)\right\|+\frac{b \Delta+c W+d W \Delta}{1-e^{-\mu \beta}}$

The above inequality shows that the value of the norm of the observation error after each location observer transition is upper bounded by an exponential with rate $-\mu$ that converges to the value $\frac{b \Delta+c W+d W \Delta}{1-e^{-\mu D}}$. Substituting the upper bound (46) into (35) and using (27), we have

$$
\begin{aligned}
& \|\zeta(t)\| \leq k\left(F_{j}\right) e^{\alpha\left(F_{j}\right)\left(t-\hat{t}_{k-1}\right)} e^{-\mu\left(\hat{t}_{k-1}-\hat{t}_{K}\right)}\left\|\zeta\left(\hat{t}_{K}\right)\right\|+k\left(F_{j}\right) e^{\alpha\left(F_{j}\right)\left(t-\hat{t}_{k-1}\right)} \frac{b \Delta+c W+d W \Delta}{1-e^{-\mu \beta}}+\sqrt{n}\left\|e^{F_{j} t}\right\|_{1} W \\
& \leq k\left(F_{j}\right) e^{-\mu\left(t-\hat{t}_{K}\right)}\left\|\zeta\left(\hat{t}_{K}\right)\right\|+\frac{k\left(F_{j}\right) b}{1-e^{-\mu \beta}} \Delta+\frac{k\left(F_{j}\right) d}{1-e^{-\mu \beta}} W \Delta+\left(\frac{k\left(F_{j}\right) c}{1-e^{-\mu \beta}}+\sqrt{n}\left\|e^{F_{j} t}\right\|_{1}\right) W \quad \forall t \in\left[\hat{t}_{k-1}, t_{k}\right)
\end{aligned}
$$

Moreover, substituting (46) into (36), we have

$$
\begin{aligned}
&\|\zeta(t)\| \leq k\left(F_{j}\right) e^{\alpha\left(F_{j}\right)\left(t-\hat{t}_{k-1}\right)} e^{-\mu\left(\hat{t}_{k-1}-\hat{t}_{K}\right)}\left\|\zeta\left(\hat{t}_{K}\right)\right\|+k\left(F_{j}\right) e^{\alpha\left(F_{j}\right)\left(t-\hat{t}_{k-1}\right)} \frac{b \Delta+c W+d W \Delta}{1-e^{-\mu \beta}} \\
&+\sqrt{n}\left\|e^{F_{j} t}\right\|_{1} W+k\left(F_{j}\right) e^{\alpha\left(F_{j}\right)\left(t-t_{k}\right)}\left(\left\|R_{j i}^{0}\right\|+\left\|I-R_{j i}^{1}\right\| B_{x}\right)+\sqrt{n} k\left(F_{j}\right) V_{j i} \Delta \\
& \leq k\left(F_{j}\right) e^{-\mu\left(t-\hat{t}_{K}\right)}\left\|\zeta\left(\hat{t}_{K}\right)\right\|+k\left(F_{j}\right)\left\{\frac{b}{1-e^{-\mu \beta}}+\sqrt{n} V_{j i}\right\} \Delta+\left(\frac{k\left(F_{j}\right) c}{1-e^{-\mu \beta}}+\sqrt{n}\left\|e^{F_{j} t}\right\|_{1}\right) W \\
&+\frac{k\left(F_{j}\right) d}{1-e^{-\mu \beta}} W \Delta+k\left(F_{j}\right)\left(\left\|R_{j i}^{0}\right\|+\left\|I-R_{j i}^{1}\right\| B_{x}\right) \quad \forall t \in\left[t_{k}, \hat{t}_{k}\right)
\end{aligned}
$$

An upper bound for the evolution of $\zeta(t)$, for $t \geq \hat{t}_{K}$ and any switching sequence of plant locations, is now obtained comparing the expressions (47) and (48):

$$
\|\zeta(t)\| \leq c_{0} e^{-\mu\left(t-t_{K}\right)}\left\|\zeta\left(t_{K}\right)\right\|+b_{3} \Delta+b_{2} W+b_{1} W \Delta+b_{0}
$$

where $c_{0}=\max _{\left\{q_{j}\right\} \in P}\left\{k\left(F_{j}\right)\right\}$,

$$
\begin{gathered}
b_{3}=\max _{\left\{q_{j}\right\} \in P} \max _{q_{i} \in \operatorname{Reach}\left(q_{j}\right)}\left\{k\left(F_{j}\right)\left(\frac{b}{1-e^{-\mu \beta}}+\sqrt{n} V_{j i}\right)\right\}, \quad b_{2}=\max _{\left\{q_{j}\right\} \in P}\left\{\frac{k\left(F_{j}\right) c}{1-e^{-\mu \beta}}+\sqrt{n}\left\|e^{F_{j} t}\right\|_{1}\right\} \\
b_{1}=\max _{\left\{q_{j}\right\} \in E_{\mathcal{O}}}\left\{\frac{k\left(F_{j}\right) d}{1-e^{-\mu \beta}}\right\}, \quad b_{0}=\max _{\left\{q_{j}\right\} \in P} \max _{q_{i} \in \operatorname{Reach}\left(q_{j}\right)}\left\{k\left(F_{j}\right)\left(\left\|R_{j i}^{0}\right\|+\left\|I-R_{j i}^{1}\right\| B_{x}\right)\right\}
\end{gathered}
$$

This proves exponentially ultimately boundedness of the hybrid observer to the ultimate bound $b_{3} \Delta+b_{2} W+b_{1} W \Delta+b_{0}$ with rate of convergence $\mu$. By choosing $\Delta$ small enough, the minimum lower bound $b_{0}+b_{2} W$ can be approached.

Finally, in the case of absence of disturbances $(W=0)$ and continuous state resets (i.e. $R_{j i}^{0}=0$ and $R_{j i}^{1}=I$ for every $\left\{q_{j}\right\} \in P$ and $\left.q_{i} \in \operatorname{Reach}\left(q_{j}\right)\right)$ any given ultimate bound can be attained by choosing an appropriate $\Delta$. 